This item was submitted to Loughborough's Research Repository by the author.

Items in Figshare are protected by copyright, with all rights reserved, unless otherwise indicated.

\title{
Sobolev weak solutions of the Hamilton--Jacobi--Bellman equations
}

PLEASE CITE THE PUBLISHED VERSION

http://dx.doi.org/10.1137/120889174

PUBLISHER

() Society for Industrial and Applied Mathematics

VERSION

AM (Accepted Manuscript)

PUBLISHER STATEMENT

This work is made available according to the conditions of the Creative Commons Attribution-NonCommercialNoDerivatives 4.0 International (CC BY-NC-ND 4.0) licence. Full details of this licence are available at: https://creativecommons.org/licenses/by-nc-nd/4.0/

\section{LICENCE}

CC BY-NC-ND 4.0

\section{REPOSITORY RECORD}

Wei, Lifeng, Zhen Wu, and Huaizhong Zhao. 2014. "Sobolev Weak Solutions of the Hamilton--jacobi--bellman Equations". Loughborough University. https://hdl.handle.net/2134/23113. 


\title{
Sobolev Weak Solution for Hamilton-Jacobi-Bellman Equation *
}

\author{
Lifeng $\mathrm{Wei}^{a} \quad$ Zhen $\mathrm{Wu}^{b} \quad$ Huaizhong Zhao ${ }^{c}$ \\ ${ }^{a}$ School of Mathematical Sciences, Ocean University of China, Qingdao 266003, P. R. China \\ E-mail address: weilifeng82@126.com (L.Wei) \\ ${ }^{b}$ School of Mathematics, Shandong University, Jinan 250100, P. R. China \\ Corresponding author. E-mail address: wuzhen@sdu.edu.cn (Z.Wu) \\ ${ }^{c}$ Department of Mathematical Sciences, Loughborough University, Loughborough, LE11 3TU, UK \\ E-mail address: h.zhao@lboro.ac.uk (H.Zhao)
}

\begin{abstract}
This paper is concerned with Sobolev weak solution of Hamilton-Jacobi-Bellman (HJB) equation. This equation is derived from the dynamic programming principle in the study of the stochastic optimal control problem. Adopting Doob-Meyer decomposition theorem as one of main tool, we prove that the optimal value function is the unique Sobolev weak solution of the corresponding HJB equation. For the recursive optimal control problem, cost function is described by the solution of backward stochastic differential equation (BSDE). This has practical background in economics and finance. We also prove that the value function is the unique Sobolev weak solution of the related HJB equation by virtue of the nonlinear Doob-Meyer decomposition theorem introduced in the BSDE theory.
\end{abstract}

Keywords: Dynamic programming principle; Hamilton-Jacobi-Bellman equation; Sobolev weak solution; backward stochastic differential equation; Doob-Meyer decomposition theorem.

\section{Introduction}

We are concerned with the classical stochastic control system governed by a nonlinear stochastic differential equation (SDE)

$$
\left\{\begin{array}{l}
d x_{s}^{t, x ; v}=b\left(s, x_{s}^{t, x ; v}, v_{s}\right) d s+\sigma\left(s, x_{s}^{t, x ; v}, v_{s}\right) d W_{s}, \quad s \in[t, T] \\
x_{t}^{t, x ; v}=x
\end{array}\right.
$$

where $b$ and $\sigma$ are given under some assumptions, $x$ is the initial data, and $W=\left(W_{t}\right)_{t \geq 0}$ is a standard $d$-dimensional Brownian motion, defined on a filtered probability space $\left(\Omega, \mathcal{F},\left(\mathcal{F}_{t}\right)_{t \geq 0}, P\right)$ satisfying the usual conditions.

The control variable $v=\left(v_{t}\right)$ is an $\mathcal{F}_{t}$ adapted process with values in some set $U$ of $R^{k}$. We denote by $\mathcal{U}$ the class of all admissible controls under some assumptions.

The cost functional is defined from $\mathcal{U}$ into $R$ by

$$
J(t, x ; v)=E\left[\int_{t}^{T} h\left(s, x_{s}^{v}, v_{s}\right) d s+g\left(x_{T}^{v}\right)\right],
$$

${ }^{*}$ This work was supported by the Natural Science Foundations of China (No. 10921101, 61174092) and the National Science Fund for Distinguished Young Scholars of China (No. 11125102). 
where $h$ and $g$ are given maps and $x_{t}^{v}$ is the trajectory of the system corresponding to $v$.

Then the value function is defined by

$$
\left\{\begin{array}{l}
V(t, x)=\sup _{v \in \mathcal{U}} J(t, x ; v), \\
V(T, x)=g(x) .
\end{array}\right.
$$

This kind of stochastic control problems have been studied extensively, both by the dynamic programming approach and by the Pontryagin stochastic maximum principle. In our paper, we are concerned with the dynamic programming principle from which we can deduce the related HJB equation. It is well known that the value function in (3) is the unique viscosity solution of the corresponding Hamilton-Jacobi-Bellman equation

$$
\left\{\begin{array}{l}
\frac{\partial u}{\partial t}+\sup _{v \in U}\left\{\frac{1}{2} \operatorname{tr}\left[\sigma \sigma^{T} \frac{\partial^{2} u}{\partial x^{2}}(t, x)\right]+\left\langle\frac{\partial u}{\partial x}, b(t, x, v)\right\rangle+h(t, x, v)\right\}=0, \\
(t, x) \in[0, T) \times R^{n} \\
\left.u\right|_{t=T}=g(x), \quad x \in R^{n} .
\end{array}\right.
$$

There are many works concerning this subject, please refer to Yong and Zhou [17] and references therein for details.

It is noted that the cost function $J$ in (2) can be regarded as the solution of one special BSDE whose generator $h$ doesnot contain solution variables $y$ and $z$. Nonlinear backward stochastic differential equation (BSDE in short) has been introduced by Pardoux and Peng [12]. Independently, Duffie and Epstein [4] introduced BSDE from economic background. In [4] they presented a stochastic differential recursive utility which is an extension of the standard additive utility with the instantaneous utility depending not only on the instantaneous consumption rate but also on the future utility. Then El Karoui, Peng and Quenez [7] gave the formulation of recursive utilities and their properties from the BSDE point of view. The recursive optimal control problem is presented as a kind of optimal control problem whose cost functional is described by the solution of BSDE. In 1992, Peng [13] got the Bellman's dynamic programming principle for this kind of problem and proved that the value function is a viscosity solution of one kind of quasi-linear second-order partial differential equation (PDE in short) which is the well-known as HJB equation. Later in 1997, he virtually generalized these results to a much more general situation, under Markvian and even non-Markvian framework (see [14]). And then $\mathrm{Wu}$ and $\mathrm{Yu}[16]$ study one kind of stochastic recursive optimal control problem with the obstacle constraint for the cost functional described by the solution of a reflected backward stochastic differential equation. They gave the dynamic programming principle for this kind of optimal control problem and show that the value function is the unique viscosity solution of the obstacle problem for the corresponding HJB equation.

We notice that all above weak solution results for HJB equations are viscosity solution results. In this paper, we will study the another kind of weak solution for this kind of HJB equations in a Sobolev space. In Section 2, some definitions and assumptions are given. And then in Section 3, we first give the definition of Sobolev weak solution for the HJB equation, and then, we prove that the value function defined by the stochastic optimal control problem is the unique Sobolev weak solution. As we know, when HJB equations are related to BSDEs for the probabilistic interpretation, the viscosity solution definition mainly corresponds to only $Y$, part of the solution of the BSDEs has an interpretation with the solution of the HJB equation, the connection of $Z$ part with the HJB equation was not given. In our Sobolev space weak 
solution definition, the interpretation for related BSDEs solution part $Z$ is more natural. One of the main tools, we adopt in this section is the Doob-Meyer decomposition theorem. To our knowledge, this is the first attempt to study Sobolev weak solution for HJB equations, it has practical importance and enrich HJB PDEs theory. In the last section, we study the Sobolev weak solution of the HJB equation corresponding to the stochastic recursive optimal control problem. We also give the definition of Sobolev weak solution for this HJB equation, and prove that the value function defined by the stochastic recursive optimal control problem is the unique Sobolev weak solution by virtue of one kind of nonlinear Doob-Meyer decomposition theorem introduced in BSDEs theory by Peng in [15].

\section{Preliminaries and Assumptions}

Let $(\Omega, \mathcal{F}, P)$ be a complete probability space, and $W=\left(W_{t}\right)_{0 \leq t \leq T}$ be a $n$-dimensional Brownian motion defined on the finite interval $[0, T], 0<T<\infty$. Denote by $\left(\mathcal{F}_{t}\right)_{0 \leq t \leq T}$ the filtration generated by the Brownian motion $W$ augmented with all $P$-null sets of $\mathcal{F}$, let $\mathcal{N}$ denote the class of $P$-null sets of $\mathcal{F}$. Let $\mathcal{F}_{t^{\prime}}^{t}$ be the complete filtration generated by the Brownian motion $W_{t^{\prime}}-W_{t}$, so $\mathcal{F}_{t^{\prime}}^{t}=\sigma\left\{W_{r}-W_{t}, t \leq r \leq t^{\prime}\right\} \vee \mathcal{N}$.

$$
\begin{aligned}
\text { Set: } & =\left\{\xi \mid \text { an } \mathcal{F}_{T}-\text { measurable random variable s.t. } E\left(|\xi|^{2}\right)<+\infty\right\}, \\
L^{2} & =\left\{\left\{\psi_{t}, 0 \leq t \leq T\right\} \mid \text { a progressively measurable process s.t. } E \int_{0}^{T}\left|\psi_{t}\right|^{2} d t<+\infty\right\}, \\
S^{2} & =\left\{\left\{\varphi_{t}, 0 \leq t \leq T\right\} \mid \text { a progressively measurable process s.t. } E\left(\sup _{0 \leq t \leq T}\left|\varphi_{t}\right|^{2}\right)<+\infty\right\} .
\end{aligned}
$$

Let $T$ be a strictly positive real number and $U$ a nonempty compact set of $R^{k}$.

In the optimal control problem, we have:

Definition 2.1 An admissible control is an $\mathcal{F}_{t}$-adapted process $v=\left(v_{t}\right)$ with values in $U$ such that

$$
E\left[\sup _{0 \leq t \leq T}\left|v_{t}\right|^{2}\right]<\infty .
$$

We denote by $\mathcal{U}$ the set of all admissible controls.

For any $v \in \mathcal{U}$, we consider the control system (1), the cost functional is defined by (2), and the value function is defined by (3), where $b, \sigma, h$ and $g$ satisfy the following assumptions:

(H1): The weight function $\rho$ is a continuous positive function on $R^{d}$ such that $\int_{R^{d}} \rho(x) d x=1$ and $\int_{R^{d}}|x|^{2} \rho(x) d x<\infty$. For instance $\rho(x)=(1+|x|)^{q}, q \leq-2$.

Denote by $L^{2}\left(R^{d}, \rho(x) d x\right)$ the weighted $L^{2}$-space with weight function endowed with the norm

$$
\|u\|_{L^{2}\left(R^{d}, \rho\right)}=\left[\int_{R^{d}}|u(x)|^{2} \rho(x) d x\right]^{\frac{1}{2}} .
$$

- We set $D:=\left\{u: R^{d} \rightarrow R\right.$ such that $u \in L^{2}\left(R^{d}, \rho(x) d x\right)$ and $\left.\frac{\partial u}{\partial x_{i}} \in L^{2}\left(R^{d}, \rho(x) d x\right)\right\}$, where 
$\frac{\partial u}{\partial x_{i}}$ denotes the derivative in the weak sense. Equipped with the norm

$$
\|u\|_{D}=\left[\int_{R^{d}}|u(x)|^{2} \rho(x) d x+\sum_{1 \leq i \leq d} \int_{R^{d}}\left|\frac{\partial u}{\partial x_{i}}\right|^{2} \rho(x) d x\right]^{\frac{1}{2}} .
$$

It is well known that $D$ is a Hilbert space, which is a classical Dirichlet space. Moreover, $D$ is a subset of the Sobolev space $H_{1}\left(R^{d}\right)$.

- We set $H:=\left\{u \in L^{2}\left(R^{d}, \rho(x) d x\right)\right.$ and $\left.\left(\sigma^{*} \nabla u\right) \in L^{2}\left(R^{d}, \rho(x) d x\right)\right\}$ equipped with the norm

$$
\|u\|_{H}=\left[\int_{R^{d}}|u(x)|^{2} \rho(x) d x+\int_{R^{d}}\left|\left(\sigma^{*} \nabla u(x)\right)\right|^{2} \rho(x) d x\right]^{\frac{1}{2}} .
$$

- We say $u \in L^{2}([0, T], H)$ iff $\int_{0}^{T}\|u(t)\|_{H}^{2} d t<\infty$.

(H2): The coefficient $b$ is 2 times continuously differentiable in $x$ and all their partial derivatives are uniformly bounded, $\sigma$ is 3 times continuously differentiable in $x$ and all their partial derivatives are uniformly bounded, and $|b(t, x, v)|+|\sigma(t, x, v)| \leq K(1+|x|)$, where $K$ is a constant.

(H3): The function $g \in L^{2}\left(R^{d}, \rho(x) d x\right)$.

(H4): The function $h:[0, T] \times R^{d} \times U \rightarrow R^{d}$ is measurable in $(t, x, v)$,

$$
E \int_{0}^{T}\left|h\left(r, 0, v_{r}\right)\right|^{2} d r \leq M
$$

and

$$
\left|h(t, x, v)-h\left(t, x^{\prime}, v\right)\right|+\left|g(x)-g\left(x^{\prime}\right)\right| \leq L\left(\left|x-x^{\prime}\right|\right) .
$$

\section{Sobolev weak solution for the HJB equation}

To study the Sobolev weak solution, we first need some norm equivalence result: SDE

For any $v \in \mathcal{U}$, consider the diffusion process $\left(x_{s}^{t, x, v}, t \leq s \leq T\right)$ which is the solution of the

$$
\left\{\begin{array}{l}
d x_{s}^{t, x ; v}=b\left(s, x_{s}^{t, x ; v}, v_{s}\right) d s+\sigma\left(s, x_{s}^{t, x ; v}, v_{s}\right) d W_{s}, \quad s \in[t, T], \\
x_{t}^{t, x ; v}=x
\end{array}\right.
$$

is a stochastic flow, and the inverse flow is denoted by $\widehat{x}_{s}^{t, x, v}$. It's known from [5] that $x \rightarrow \widehat{x}_{s}^{t, x, v}$ is differentiable and we denote by $J\left(\widehat{x}_{s}^{t, x, v}\right)$ the determinant of the Jacobian matrix of $\widehat{x}_{s}^{t, x, v}$, which is positive and $J\left(\widehat{x}_{t}^{t, x, v}\right)=1$. For $\varphi \in C_{c}^{\infty}\left(R^{d}\right)$ we define a process $\varphi_{t}: \Omega \times[0, T] \times R^{d} \rightarrow R$ by $\varphi_{t}(s, x)=\varphi\left(\widehat{x}_{s}^{t, x, v}\right) J\left(\widehat{x}_{s}^{t, x, v}\right)$. Following Kunita [6], we can define the composition of $u \in L^{2}\left(R^{d}\right)$ with the stochastic flow by $\left(u \circ x_{s}^{t, \cdot, v}, \varphi\right)=\left(u, \varphi_{t}(s, \cdot)\right)$. Indeed, by a change of variable, we have

$$
\left(u \circ x_{s}^{t, \cdot, v}, \varphi\right)=\int_{R^{d}} u(y) \varphi\left(\widehat{x}_{s}^{t, x, v}\right) J\left(\widehat{x}_{s}^{t, x, v}\right) d y=\int_{R^{d}} u\left(x_{s}^{t, x, v}\right) \varphi(x) d x .
$$

In [1], V. Bally and A. Matoussi proved that $\varphi_{t}(s, x)$ is a semimartingale and admits the following 
Lemma 3.1 For $\varphi \in C_{c}^{2}\left(R^{d}\right)$, we have

$$
\varphi_{t}(s, x)=\varphi(x)-\sum_{j=1}^{d} \int_{t}^{s} \sum_{i=1}^{d} \frac{\partial}{\partial x_{i}}\left(\sigma_{i, j}(r, x) \varphi_{t}(r, x)\right) d W_{r}^{j}+\int_{t}^{s} L_{r}^{*} \varphi_{t}(r, x) d r,
$$

where $L_{t}^{*}$ is the adjoint operator of $L_{t}$.

The next lemma, known as the norm equivalence result and proved in [1] plays an important role in the proof of the main result.

Lemma 3.2 Let $\rho$ be a weight function defined and assume (H1) and (H2) holds, for any $v \in \mathcal{U}$ then there exists two constants $c>0$ and $C>0$ such that for every $t \leq s \leq T$ and $\varphi \in L^{1}\left(R^{d} ; \rho(x) d x\right)$,

$$
c \int_{R^{d}}|\varphi(x)| \rho(x) d x \leq \int_{R^{d}} E\left(\left|\varphi\left(x_{s}^{t, x ; v}\right)\right|\right) \rho(x) d x \leq C \int_{R^{d}}|\varphi(x)| \rho(x) d x .
$$

Moreover, for every $\psi \in L^{1}\left([0, T] \times R^{d} ; d t \otimes \rho(x) d x\right)$,

$c \int_{R^{d}} \int_{t}^{T}|\psi(s, x)| d s \rho(x) d x \leq \int_{R^{d}} \int_{t}^{T} E\left(\left|\psi\left(s, x_{s}^{t, x ; v}\right)\right|\right) d s \rho(x) d x \leq C \int_{R^{d}} \int_{t}^{T}|\psi(s, x)| d s \rho(x) d x$.

The constants $c$ and $C$ depend on $T$, on $\rho$ and on the bounds of derivatives of the $b$ and $\sigma$. The proof is similar to the proof of Proposition 5.1 in [1], hence we omit it.

Now, we first give the definition of a Sobolev solution for HJB equation (4).

Definition 3.1 We say that $V$ is a weak solution of HJB equation (4), if

(i) $V \in L^{2}([0, T] ; H)$, i.e.,

$$
\int_{0}^{T}\|V(t)\|_{H}^{2} d t=\int_{0}^{T}\left(\int_{R^{d}}|V(t, x)|^{2} \rho(x) d x+\int_{R^{d}}\left|\left(\sigma^{*} \nabla V\right)(t, x)\right|^{2} \rho(x) d x\right) d t<\infty .
$$

(ii) For any nonnegative $\varphi \in C_{c}^{1, \infty}\left([0, T] \times R^{d}\right)$ and for any $v \in \mathcal{U}$, then

$$
\begin{aligned}
& \int_{R^{d}} \int_{s}^{T}\left(V(r, x), \partial_{r} \varphi(r, x)\right) d r d x+\int_{R^{d}}(V(s, x), \varphi(s, x)) d x \\
\geq & \int_{R^{d}}(g(x), \varphi(T, x)) d x+\int_{R^{d}} \int_{s}^{T}(h(r, x, v), \varphi(r, x)) d r d x \\
& +\int_{R^{d}} \int_{s}^{T}\left(\mathcal{L}_{r}^{v} V(r, x), \varphi(r, x)\right) d r d x,
\end{aligned}
$$

where $\left(L_{r} V(r, x), \varphi(r, x)\right)=\int_{R^{d}}\left(\frac{1}{2}(\nabla V \sigma)\left(\sigma^{*} \nabla \varphi\right)+V \operatorname{div}(b-A) \varphi\right) d x$ with $A_{i}=\frac{1}{2} \sum_{k=1}^{d} \frac{\partial a_{k, i}}{\partial x_{k}}$.

(iii) For any nonnegative $\varphi \in C_{c}^{1, \infty}\left([0, T] \times R^{d}\right)$ and for any small $\varepsilon>0$, there exists a control $v^{\prime} \in \mathcal{U}$, such that

$$
\begin{aligned}
& \int_{R^{d}} \int_{s}^{T}\left(V(r, x), \partial_{r} \varphi(r, x)\right) d r d x+\int_{R^{d}}(V(s, x), \varphi(s, x)) d x-\varepsilon \\
\leq & \int_{R^{d}}(g(x), \varphi(T, x)) d x+\int_{R^{d}} \int_{s}^{T}(h(r, x, v), \varphi(r, x)) d r d x \\
& +\int_{R^{d}} \int_{s}^{T}\left(\mathcal{L}_{r}^{v^{\prime}} V(r, x), \varphi(r, x)\right) d r d x .
\end{aligned}
$$


From the Bellman's dynamic programming principle (Theorem 3.3, Chapter 4 in Yong and Zhou [17] and Theorem 6.1 in [14] for non-Markov case), we can prove:

Lemma 3.3 Let (H1)-(H4) hold, then for any $v \in \mathcal{U}$, the value function satisfies

$$
V\left(s, x_{s}^{t, x, v}\right) \geq E\left\{\int_{s}^{s^{\prime}} h\left(r, x_{r}^{t, x, v}, v_{r}\right) d r+V\left(s^{\prime}, x_{s^{\prime}}^{t, x, v}\right) \mid \mathcal{F}_{s}^{t}\right\}, \quad \forall t \leq s \leq s^{\prime} \leq T .
$$

For any small $\varepsilon>0$, there exists a $v^{\prime} \in \mathcal{U}$, such that

$$
V\left(s, x_{s}^{t, x, v^{\prime}}\right)-\varepsilon \leq E\left\{\int_{s}^{s^{\prime}} h\left(r, x_{r}^{t, x, v^{\prime}}, v_{r}^{\prime}\right) d r+V\left(s^{\prime}, x_{s^{\prime}}^{t, x, v^{\prime}}\right) \mid \mathcal{F}_{s}^{t}\right\} \quad \forall t \leq s \leq s^{\prime} \leq T .
$$

Proof: We adopt the semigroup notation $G(\cdot)$ from [16] and [14], and get the generalized dynamic programming principle:

$$
V\left(s, x_{s}^{t, x, v}\right)=e s s \sup _{v \in \mathcal{U}} G_{s, s^{\prime}}^{t, x, v}\left[V\left(s^{\prime}, x_{s^{\prime}}^{t, x, v}\right)\right], \quad \forall t \leq s \leq s^{\prime} \leq T
$$

here we denote

$$
G_{s, s^{\prime}}^{t, x, v}\left[V\left(s^{\prime}, x_{s^{\prime}}^{t, x, v}\right)\right]:=\widetilde{y}_{s}^{t, x, v},
$$

one solution of the BSDE satisfying

$$
\widetilde{y}_{s}^{t, x, v}=V\left(s^{\prime}, x_{s^{\prime}}^{t, x, v}\right)+\int_{s}^{s^{\prime}} h\left(r, x_{r}^{t, x, v}, v_{r}\right) d r-\int_{s}^{s^{\prime}} \widetilde{z}_{r}^{t, x, v} d W_{r},
$$

i.e.

$$
\widetilde{y}_{s}^{t, x, v}=E\left\{\int_{s}^{s^{\prime}} h\left(r, x_{r}^{t, x, v}, v_{r}\right) d r+V\left(s^{\prime}, x_{s^{\prime}}^{t, x, v}\right) \mid \mathcal{F}_{s}^{t}\right\}
$$

then it is easy to get (8) and (9).

Then we continue to have

Lemma 3.4 Let (H1)-(H4) hold, for any $v$, there exists a unique increasing process $\left(A_{r}\right)$ with $A_{t}=0$ and $E\left[\left(A_{T}\right)^{2}\right]<\infty$ such that $V\left(s, x_{s}^{t, x, v}\right)$ coincides with the unique solution $y_{s}^{t, x, v}$ of the $B S D E$ :

$$
y_{t}^{t, x, v}=V\left(T, x_{T}^{t, x, v}\right)+\int_{t}^{T} h\left(r, x_{r}^{t, x, v}, v_{r}\right) d r+A_{T}^{t, x, v}-A_{t}^{t, x, v}-\int_{t}^{T} Z_{r}^{t, x, v} d W_{r},
$$

where $Z_{r}^{t, x, v}=\sigma^{*} \nabla V\left(r, x_{r}^{t, x, v}\right)$ in the sense of Definition 3.1 .

Proof: By the inequality (8) in Lemma 3.3, we know that for $t \leq s \leq t^{\prime}$,

$$
\begin{aligned}
& V\left(s, x_{s}^{t, x, v}\right)+\int_{t}^{s} h\left(r, x_{r}^{t, x, v}, v_{r}\right) d r \\
\geq & E\left\{V\left(t^{\prime}, x_{t^{\prime}}^{t, x, v}\right)+\int_{t}^{t^{\prime}} h\left(r, x_{r}^{t, x, v}, v_{r}\right) d r \mid \mathcal{F}_{s}^{t}\right\},
\end{aligned}
$$


so $V\left(s, x_{s}^{t, x, v}\right)+\int_{t}^{s} h\left(r, x_{r}^{t, x, v}, v_{r}\right) d r$ is a supermartingale. By the Doob-Meyer decomposition theorem (Theorem 3.8, Chapter 3 in [5]), we know that there exists an increasing process $A^{t, x, v}$ satisfying

$$
\begin{aligned}
& V\left(t^{\prime}, x_{t^{\prime}}^{t, x, v}\right)+\int_{t}^{t^{\prime}} h\left(r, x_{r}^{t, x, v}, v_{r}\right) d r \\
= & V\left(s, x_{s}^{t, x, v}\right)+\int_{t}^{s} h\left(r, x_{r}^{t, x, v}, v_{r}\right) d r+\int_{s}^{t^{\prime}} Z_{r}^{t, x, v} d W_{r}-\left(A_{t^{\prime}}^{t, x, v}-A_{s}^{t, x, v}\right) .
\end{aligned}
$$

So

$$
V\left(s, x_{s}^{t, x, v}\right)=V\left(t^{\prime}, x_{t^{\prime}}^{t, x, v}\right)+\int_{s}^{t^{\prime}} h\left(r, x_{r}^{t, x, v}, v_{r}\right) d r-\int_{s}^{t^{\prime}} Z_{r}^{t, x, v} d W_{r}+\left(A_{t^{\prime}}^{t, x, v}-A_{s}^{t, x, v}\right) .
$$

We consider the following family of BSDE parameterized by $i=1,2, \cdots$

$$
y_{t}^{t, x, v ; i}=V\left(T, x_{T}^{t, x, v}\right)+\int_{t}^{T} h\left(r, x_{r}^{t, x, v}, v_{r}\right) d r+i \int_{t}^{T}\left(V\left(r, x_{r}^{t, x, v}\right)-y_{r}^{t, x, v ; i}\right) d r-\int_{t}^{T} Z_{r}^{t, x, v ; i} d W_{r} .
$$

We let $t^{\prime}=T$ in (17) and compare (17) with (18), it is easy to know that $V\left(r, x_{r}^{t, x, v}\right)-y_{r}^{t, x, v ; i} \geq 0$. And then, noting (18), it follows from the comparison theorem of the BSDE, that $y_{r}^{t, x, v ; i} \leq$ $y_{r}^{t, x, v ; i+1}$. Thus $\left\{y_{r}^{t, x, v ; i}\right\}$ is monotonically converges up to a process $y_{r}^{t, x, v}$, moreover $y_{r}^{t, x, v}$ is bounded by $V\left(r, x_{r}^{t, x, v}\right)$. We desire to prove this process $y_{r}^{t, x, v}$ satisfies (14).

We denote

$$
A_{r}^{t, x, v ; i}:=i \int_{t}^{r}\left(V\left(s, x_{s}^{t, x, v}\right)-y_{s}^{t, x, v ; i}\right) d s .
$$

From BSDE (18), we have

$$
\begin{aligned}
A_{T}^{t, x, v ; i}-A_{t}^{t, x, v ; i}= & y_{t}^{t, x, v ; i}-V\left(T, x_{T}^{t, x, v}\right)-\int_{t}^{T} h\left(r, x_{r}^{t, x, v}, v_{r}\right) d r+\int_{t}^{T} Z_{r}^{t, x, v ; i} d W_{r} \\
\leq & \left|y_{t}^{t, x, v ; i}\right|+\left|V\left(T, x_{T}^{t, x, v}\right)\right|+\int_{t}^{T} h\left(r, 0, v_{r}\right) d r+\int_{t}^{T}\left(L\left|x_{r}^{t, x, v}\right|\right) d r \\
& +\left|\int_{t}^{T} Z_{r}^{t, x, v, i} d W_{r}\right| \\
\leq & |V(t, x)|+\left|V\left(T, x_{T}^{t, x, v}\right)\right|+\int_{t}^{T} h\left(r, 0, v_{r}\right) d r+\int_{t}^{T}\left(L\left|x_{r}^{t, x, v}\right|\right) d r \\
& +\left|\int_{t}^{T} Z_{r}^{t, x, v, i} d W_{r}\right| .
\end{aligned}
$$

For any $v_{r}$, then $E \int_{t}^{T}\left|h\left(r, 0, v_{r}\right)\right|^{2} d r \leq M$. Recall the estimate about $x_{r}^{t, x, v}$,

$$
E\left\{\sup _{t \leq r \leq T}\left|x_{r}^{t, x, v}-x\right|^{2}\right\} \leq C(T-t),
$$

and

$$
E\left\{\sup _{t \leq r \leq T}\left|x_{r}^{t, x, v}\right|^{2}\right\} \leq C_{0}\left(1+|x|^{2}\right)
$$

where constants $C$ and $C_{0}$ only depend on Lipschiz Constants of $b$ and $\sigma$. Then

$$
E \int_{t}^{T}\left|x_{r}^{t, x, v}\right|^{2} d r \leq C_{0}\left(1+|x|^{2}\right)(T-t) .
$$


So we can get

$$
\begin{aligned}
& E\left|A_{T}^{t, x, v ; i}\right|^{2} \\
\leq & 5|V(t, x)|^{2}+5|g(0)|^{2}+5 E \int_{t}^{T}\left|h\left(r, 0, v_{r}\right)\right|^{2} d r+5 E \int_{t}^{T}\left(L\left|x_{r}^{t, x, v}\right|^{2}\right) d r \\
& +5 E \int_{t}^{T}\left|Z_{r}^{t, x, v, i}\right|^{2} d r \\
\leq & C_{1}(t, T, x, v)+5 E \int_{t}^{T}\left|Z_{r}^{t, x, v, i}\right|^{2} d r .
\end{aligned}
$$

where $C_{1}(t, T, x, v)=5|V(t, x)|^{2}+5|g(0)|^{2}+5 M+5 C_{0}\left(1+|x|^{2}\right)(T-t)$ is independent of $i$. On the other hand, we use the Itô's formula applied to $\left|y_{r}^{t, x, v ; i}\right|^{2}$ :

$$
\begin{aligned}
& \left|y_{t}^{t, x, v ; i}\right|^{2}+E \int_{t}^{T}\left|Z_{r}^{t, x, v ; i}\right|^{2} d r \\
= & E\left|V\left(T, x_{T}^{t, x, v}\right)\right|^{2}+2 E \int_{t}^{T} y_{r}^{t, x, v ; i} h\left(r, x_{r}^{t, x, v}, v_{r}\right) d r+2 E \int_{t}^{T} y_{r}^{t, x, v ; i} d A_{r}^{t, x, v ; i} \\
\leq & E\left|V\left(T, x_{T}^{t, x, v}\right)\right|^{2}+E \int_{t}^{T}\left|y_{r}^{t, x, v ; i}\right|^{2} d r+L^{2} E \int_{t}^{T}\left|x_{r}^{t, x, v}\right|^{2} d r \\
& +E \int_{t}^{T}\left|h\left(r, 0, v_{r}\right)\right|^{2} d r+2 E\left[A_{T}^{t, x, v ; i} \sup _{t \leq s \leq T}\left|y_{s}^{t, x, v ; i}\right|\right] \\
\leq & E\left|V\left(T, x_{T}^{t, x, v}\right)\right|^{2}+E \int_{t}^{T}\left|y_{r}^{t, x, v ; i}\right|^{2} d r+L^{2} E \int_{t}^{T}\left|x_{r}^{t, x, v}\right|^{2} d r+E \int_{t}^{T}\left|h\left(r, 0, v_{r}\right)\right|^{2} d r \\
& +\frac{1}{10} E\left[\left|A_{T}^{t, x, v ; i}\right|^{2}\right]+10 E\left[\sup _{t \leq s \leq T}\left|y_{s}^{t, x, v ; i}\right|^{2}\right],
\end{aligned}
$$

by the property of the $V(t, x)$ (see lemma 6.3 in [14] or Proposition 3.1 of Chapter 4 in [17]), $E\left|V\left(s, x_{s}^{t, x, v}\right)-V(s, x)\right|^{2} \leq E\left|x_{s}^{t, x, v}-x\right|^{2} \leq C(T-t)$ and $y_{r}^{t, x, v, i} \leq V\left(r, x_{r}^{t, x, v}\right)$. We can get

$$
E \int_{t}^{T}\left|y_{r}^{t, x, v ; i}\right|^{2} d r+10 E\left[\sup _{t \leq s \leq T}\left|y_{s}^{t, x, v ; i}\right|^{2}\right] \leq E \int_{t}^{T}\left|V\left(r, x_{r}^{t, x, v}\right)\right|^{2} d r+10 E\left[\sup _{t \leq s \leq T}\left|V\left(s, x_{s}^{t, x, v}\right)\right|^{2}\right] .
$$

So we also can define a $C_{2}(t, T, x, v)$ satisfying

$$
E \int_{t}^{T}\left|Z_{r}^{t, x, v ; i}\right|^{2} d r \leq C_{2}(t, T, x, v)+\frac{1}{10} E\left[\left|A_{T}^{t, x, v ; i}\right|^{2}\right] .
$$

Here $C_{1}, C_{2}$ are independent of $i$, so we can get

$$
E\left[\left|A_{T}^{t, x, v ; i}\right|^{2} \leq 2 C_{1}(t, T, x, v)+10 C_{2}(t, T, x, v) .\right.
$$

Then

$$
i^{2} E \int_{t}^{r}\left(V\left(s, x_{s}^{t, x, v}\right)-y_{s}^{t, x, v ; i}\right)^{2} d s \leq 2 C_{1}(t, T, x, v)+10 C_{2}(t, T, x, v),
$$

we let $i \rightarrow \infty$, it also holds. Now applying Fatou's Lemma, we can get $E \int_{t}^{r}\left(V\left(s, x_{s}^{t, x, v}\right)-\right.$ $\left.y_{s}^{t, x, v ; i}\right)^{2} d s=0$, so $y_{r}^{t, x, v}=V\left(r, x_{r}^{t, x, v}\right)$ for any $r \in[t, T]$ a.s., and satisfies the following BSDE:

$$
y_{r}^{t, x, v}=V\left(T, x_{T}^{t, x, v}\right)+\int_{r}^{T} h\left(s, x_{s}^{t, x, v}, v_{s}\right) d s+\left(A_{T}-A_{r}\right)-\int_{r}^{T} Z_{s}^{t, x, v} d W_{s},
$$


where $A_{t}$ is a RCLL increasing process.

Now we look back BSDE (18) again, we have $i\left(V\left(r, x_{r}^{t, x, v}\right)-y_{r}^{t, x, v ; i}\right)$ is Lipschitz about $y_{r}^{t, x, v ; i}$, and

$$
\int_{t}^{T} \int_{R^{d}}\left(\left|h\left(r, 0, v_{r}\right)\right|^{2}+|V(r, x)|^{2}\right) \rho(x) d x d r<\infty .
$$

From the fact that $b, \sigma$ satisfy the (H2), so by the Lemma 4.1 in [10] or Theorem 3.1 in [1], we know $Z_{r}^{t, x, v ; i}=\sigma^{*} \nabla y_{r}^{t, x, v ; i}$.

By the estimate of the BSDE, we know that there exists a $Z_{r}^{t, x, v}$ as the weak limit of $Z_{r}^{t, x, v ; i}$ in $L^{2}\left([t, T], R^{d}\right)$,

$$
\begin{aligned}
& E\left|Y_{t}^{t, x, v, m}-Y_{t}^{t, x, v, n}\right|^{2}+E \int_{t}^{T}\left|Z_{r}^{t, x, v, m}-Z_{r}^{t, x, v, n}\right|^{2} d r \\
= & E \int_{t}^{T}\left|Y_{r}^{t, x, v, m}-Y_{r}^{t, x, v, n}\right| d\left(A_{r}^{t, x, v, m}-A_{r}^{t, x, v, n}\right) \\
\leq & E \sup _{t \leq r \leq T}\left|Y_{r}^{t, x, v, m}-Y_{r}^{t, x, v, n}\right|\left(A_{T}^{t, x, v, m}-A_{T}^{t, x, v, n}\right) \\
\leq & \left(E \sup _{t \leq r \leq T}\left|Y_{r}^{t, x, v, m}-Y_{r}^{t, x, v, n}\right|^{2}\right)^{\frac{1}{2}}\left(E\left(A_{T}^{t, x, v, m}-A_{T}^{t, x, v, n}\right)^{2}\right)^{\frac{1}{2}} .
\end{aligned}
$$

Because $E\left(A_{T}^{t, x, v, i}\right)^{2}$ is bounded, and $Y_{r}^{t, x, v, m}$ is convergent, so

$$
\lim _{m, n \rightarrow \infty} E \int_{t}^{T}\left|Z_{r}^{t, x, v ; n}-Z_{r}^{t, x, v ; m}\right|^{2} d r=0
$$

then $Z_{r}^{t, x, v}=\sigma^{*} \nabla y_{r}^{t, x, v}=\sigma^{*} \nabla V\left(r, x_{r}^{t, x, v}\right)$.

Lemma 3.5 We assume (H1)-(H4) hold, and for any small $\varepsilon>0$, there exists a control $v^{\prime} \in \mathcal{U}$, such that $V\left(s, x_{s}^{t, x, v^{\prime}}\right)$ satisfies (9), i.e.

$$
V\left(s, x_{s}^{t, x, v^{\prime}}\right) \leq E\left\{\int_{s}^{s^{\prime}}\left(h\left(r, x_{r}^{t, x, v^{\prime}}, v_{r}^{\prime}\right)+\frac{\varepsilon}{T-t}\right) d r+V\left(s^{\prime}, x_{s^{\prime}}^{t, x, v^{\prime}}\right) \mid \mathcal{F}_{s}^{t}\right\}, \quad \forall 0 \leq t \leq s \leq t^{\prime} \leq T .
$$

Then there exists a unique increasing process $\left(A_{r}^{\prime}\right)$ with $A_{t}^{\prime}=0$ and $E\left[\left(A_{T}^{\prime}\right)^{2}\right]<\infty$ such that $V\left(s, x_{s}^{t, x, v^{\prime}}\right)$ coincides with the unique solution $y_{s}^{t, x, v}$ of the BSDE:

$$
y_{t}^{t, x, v^{\prime}}=V\left(T, x_{T}^{t, x, v^{\prime}}\right)+\int_{t}^{T}\left[h\left(r, x_{r}^{t, x, v^{\prime}}, v_{r}^{\prime}\right)+\frac{\varepsilon}{T-t}\right] d r-\left(A_{T}^{t, x, v^{\prime}}-A_{t}^{t, x, v^{\prime}}\right)-\int_{t}^{T} Z_{r}^{t, x, v^{\prime}} d W_{r},
$$

where $Z_{r}^{t, x, v^{\prime}}=\sigma^{*} \nabla V\left(r, x_{r}^{t, x, v^{\prime}}\right)$ in the sense of Definition 3.1 .

The proof of Lemma 3.5 is similar to Lemma 3.4, hence we omit it.

Now we give the main result of this section.

Theorem 3.1 Under the assumption (H1)-(H4), the value function $V(t, x)$ defined by (3) is the unique Sobolev solution of the PDE (4). 
Proof: Existence: In the stochastic optimal control problem $(2), V(t, x)$ defined by (3) satisfies Bellman's dynamic programming principle. By Lemmas 3.3-3.5, we know that, for any $v \in \mathcal{U}$, there exists a unique increasing process $A_{s}^{t, x, v}, V\left(s, x_{s}^{t, x, v}\right)$ satisfy the following BSDE:

$$
V\left(s, x_{s}^{t, x, v}\right)=V\left(T, x_{T}^{t, x, v}\right)+\int_{s}^{T} h\left(r, x_{r}^{t, x, v}, v_{r}\right) d r+\left(A_{T}^{t, x, v}-A_{s}^{t, x, v}\right)-\int_{s}^{T} \sigma^{*} \nabla V\left(r, x_{r}^{t, x, v}\right) d W_{r} .
$$

So, we can get

$$
V\left(s, x_{s}^{t, x, v}\right) \geq V\left(T, x_{T}^{t, x, v}\right)+\int_{s}^{T} h\left(r, x_{r}^{t, x, v}, v_{r}\right) d r-\int_{s}^{T} \sigma^{*} \nabla V\left(r, x_{r}^{t, x, v}\right) d W_{r} .
$$

On the other hand, for any small $\varepsilon>0$ there exists a control $v^{\prime} \in \mathcal{U}, V\left(s, x_{s}^{t, x, v^{\prime}}\right)$ satisfy the following BSDE:

$$
\begin{aligned}
V\left(s, x_{s}^{t, x, v^{\prime}}\right)-\varepsilon \leq & V\left(T, x_{T}^{t, x, v^{\prime}}\right)+\int_{s}^{T} h\left(r, x_{r}^{t, x, v^{\prime}}, v_{r}^{\prime}\right) d r-\left(A_{T}^{t, x, v^{\prime}}-A_{s}^{t, x, v^{\prime}}\right) \\
& -\int_{s}^{T} \sigma^{*} \nabla V\left(r, x_{r}^{t, x, v^{\prime}}\right) d W_{r} .
\end{aligned}
$$

So we can get

$$
V\left(s, x_{s}^{t, x, v^{\prime}}\right)-\varepsilon \leq V\left(T, x_{T}^{t, x, v^{\prime}}\right)+\int_{s}^{T} h\left(r, x_{r}^{t, x, v^{\prime}}, v_{r}^{\prime}\right) d r-\int_{s}^{T} \sigma^{*} \nabla V\left(r, x_{r}^{t, x, v^{\prime}}\right) d W_{r} .
$$

We can deduce by the equivalence of norm result (Lemma 3.2) that $V \in L^{2}([t, T], H)$. However, in the stochastic optimal control problem (2), the cost function $J(t, x ; v)$ can be regarded as as a solution of a BSDE $Y_{t}$, which satisfies

$$
Y_{s}^{t, x, v}=g\left(x_{T}^{t, x, v}\right)+\int_{s}^{T} h\left(r, x_{r}^{t, x, v}, v_{r}\right) d r-\int_{s}^{T} Z_{r}^{t, x, v} d W_{r}, t \leq s \leq T .
$$

By the usual estimate for the solution of the BSDE, and (H1)-(H4) we know that

$$
\begin{aligned}
& \int_{R^{d}} E\left(\sup _{t \leq r \leq T}\left|Y_{r}^{t, x, v}\right|^{2}+\int_{t}^{T}\left|Z_{r}^{t, x, v}\right|^{2} d r\right) \rho(x) d x \\
\leq & \int_{R^{d}} E\left|g\left(x_{T}^{t, x, v}\right)\right|^{2} \rho(x) d x+\int_{R^{d}} E \int_{t}^{T}\left|h\left(r, x_{r}^{t, x, v}, v_{r}\right)\right|^{2} d r \rho(x) d x \\
\leq & C \int_{R^{d}}|g(x)|^{2} \rho(x) d x+\int_{R^{d}} \int_{t}^{T}\left|h\left(r, 0, v_{r}\right)\right|^{2} d r \rho(x) d x+C \int_{R^{d}} \int_{t}^{T}|x|^{2} \rho(x) d x d t<\infty \\
\leq & C \int_{R^{d}}|g(x)|^{2} \rho(x) d x+M(T-t)+C(T-t) \int_{R^{d}}|x|^{2} \rho(x) d x<\infty .
\end{aligned}
$$

So for any $v, Y_{r}^{t, \cdot, v} \in H$, here $H$ is a Hilbert space, $V(t, x)=\sup _{v \in U} Y_{t}^{t, x, v}$. And then from $U$ is compact set of $R^{k}$ and Lemma 6.2 in [14], we know that $V(t, \cdot)$ is also in $H$.

Next because (28) holds, then for any nonnegative $\varphi \in C_{c}^{\infty}\left(R^{d}\right)$, we have

$$
\begin{aligned}
& \int_{R^{d}}\left(V\left(s, x_{s}^{t, x, v}\right), \varphi(x)\right) d x \\
\geq & \int_{R^{d}}\left(V\left(T, x_{T}^{t, x, v}\right), \varphi(x)\right) d x+\int_{R^{d}} \int_{s}^{T}\left(h\left(r, x_{r}^{t, x, v}, v_{r}\right), \varphi(x)\right) d r d x \\
& -\int_{R^{d}} \int_{s}^{T}\left(\sigma^{*} \nabla V\left(r, x_{r}^{t, x, v}\right), \varphi(x)\right) d W_{r} d x .
\end{aligned}
$$


That is

$$
\begin{aligned}
& \int_{R^{d}}(V(s, x), \varphi(s, x)) d x \\
\geq & \int_{R^{d}}(g(x), \varphi(T, x)) d x+\int_{R^{d}} \int_{s}^{T}\left(h\left(r, x, v_{r}\right), \varphi(r, x)\right) d r d x \\
& -\int_{R^{d}} \int_{s}^{T}\left(\sigma^{*} \nabla V(r, x), \varphi(r, x)\right) d W_{r} d x .
\end{aligned}
$$

Furthermore, using Lemma 3.1, we have

$$
\begin{aligned}
& -\int_{R^{d}} \int_{s}^{T} \sigma^{*} \nabla V(r, x) \varphi(r, x) d W_{r} d x \\
= & -\int_{R^{d}} \sum_{j=1}^{d} \int_{s}^{T}\left(\sum_{i=1}^{d} \sigma_{i, j}(r, x) \frac{\partial V}{\partial x_{i}}(r, x), \varphi(r, x)\right) d W_{r}^{j} d x \\
= & \int_{R^{d}} \int_{s}^{T}\left(\mathcal{L}^{v} V(r, x), \varphi(r, x)\right) d r-\int_{R^{d}} \int_{s}^{T}\left(V(r, x), \partial_{r} \varphi(r, x)\right) d r d x .
\end{aligned}
$$

Taking (35) into (34), we can get

$$
\begin{aligned}
& \int_{R^{d}} \int_{s}^{T}\left(V(r, x), \partial_{r} \varphi(r, x)\right) d r d x+\int_{R^{d}}(V(s, x), \varphi(s, x)) d x \\
\geq & \int_{R^{d}}(g(x), \varphi(T, x)) d x+\int_{R^{d}} \int_{s}^{T}\left(h\left(r, x, v_{r}\right), \varphi(r, x)\right) d r d x \\
& +\int_{R^{d}} \int_{s}^{T}\left(\mathcal{L}_{r}^{v} V(r, x), \varphi(r, x)\right) d r d x .
\end{aligned}
$$

Using the same techniques and methods, noting (30) holds, then for any nonnegative $\varphi \in$ $C_{c}^{\infty}\left(R^{d}\right)$, we take $\varepsilon=\frac{\varepsilon^{\prime}}{\int_{R^{d}} \varphi(x) d x}$, so

$$
\begin{aligned}
& \int_{R^{d}}\left(V\left(s, x_{s}^{t, x, v^{\prime}}\right), \varphi(x)\right) d x-\varepsilon^{\prime} \\
\leq & \int_{R^{d}}\left(g\left(x_{T}^{t, x, v^{\prime}}\right), \varphi(x)\right) d x+\int_{R^{d}} \int_{s}^{T}\left(h\left(r, x_{r}^{t, x, v^{\prime}}, v_{r}^{\prime}\right), \varphi(x)\right) d r d x \\
& -\int_{R^{d}} \int_{s}^{T}\left(\sigma^{*} \nabla V\left(r, x_{r}^{t, x, v^{\prime}}\right), \varphi(x)\right) d W_{r} d x,
\end{aligned}
$$

that is

$$
\begin{aligned}
& \int_{R^{d}}(V(s, x), \varphi(s, x)) d x-\varepsilon^{\prime} \\
\leq & \int_{R^{d}}(g(x), \varphi(T, x)) d x+\int_{R^{d}} \int_{s}^{T}\left(h\left(r, x, v_{r}\right), \varphi(r, x)\right) d r d x \\
& -\int_{R^{d}} \int_{s}^{T}\left(\sigma^{*} \nabla V(r, x), \varphi(r, x)\right) d W_{r} d x .
\end{aligned}
$$


Taking (35) into (37), we can get

$$
\begin{aligned}
& \int_{R^{d}} \int_{s}^{T}\left(V(r, x), \partial_{r} \varphi(r, x)\right) d r d x+\int_{R^{d}}(V(s, x), \varphi(s, x)) d x-\varepsilon^{\prime} \\
\leq & \int_{R^{d}}(g(x), \varphi(T, x)) d x+\int_{R^{d}} \int_{s}^{T}\left(h\left(r, x, v_{r}\right), \varphi(r, x)\right) d r d x \\
& +\int_{R^{d}} \int_{s}^{T}\left(\mathcal{L}_{r}^{v^{\prime}} V(r, x), \varphi(r, x)\right) d r d x .
\end{aligned}
$$

Uniqueness:

Let $\bar{V}$ be another solution of the PDE (4). By Definition 3.1, one gets: for any $v \in \mathcal{U}$,

$$
\begin{aligned}
& \int_{R^{d}} \int_{s}^{T}\left(\bar{V}(r, x), \partial_{r} \varphi(r, x)\right) d r d x+\int_{R^{d}}(\bar{V}(s, x), \varphi(s, x)) d x \\
\geq & \int_{R^{d}}(g(x), \varphi(T, x)) d x+\int_{R^{d}} \int_{s}^{T}\left(h\left(r, x, v_{r}\right), \varphi(r, x)\right) d r d x \\
& +\int_{R^{d}} \int_{s}^{T}\left(\mathcal{L}_{r}^{v} \bar{V}(r, x), \varphi(r, x)\right) d r d x .
\end{aligned}
$$

By Lemma 4.5 in [11], we have

$$
\begin{aligned}
& \int_{R^{d}} \int_{s}^{T}\left(\bar{V}(r, x), \partial_{r} \varphi(r, x)\right) d r d x \\
= & \int_{R^{d}} \Sigma_{j=1}^{d} \int_{s}^{T}\left(\Sigma_{i=1}^{d}\left(\sigma_{i, j} \frac{\partial \bar{V}}{\partial x_{i}}(r, x), \varphi(r, x)\right)\right) d W_{r} d x+\int_{R^{d}} \int_{s}^{T}\left(L_{r}^{v} \bar{V}(r, x), \varphi(r, x)\right) d r d x \\
= & \int_{R^{d}} \int_{s}^{T}\left(\left(\sigma^{*} \nabla \bar{V}\right)(r, x), \varphi(r, x)\right) d W_{r} d x+\int_{R^{d}} \int_{s}^{T}\left(L_{r}^{v} \bar{V}(r, x), \varphi(r, x)\right) d r d x .
\end{aligned}
$$

Taking (40) into (39), we can get

$$
\begin{aligned}
& \int_{R^{d}}(\bar{V}(s, x), \varphi(s, x)) d x+\int_{R^{d}} \int_{s}^{T}\left(\sigma^{*} \nabla \bar{V}\right)(r, x) \varphi(r, x) d W_{r} d x \\
& +\int_{R^{d}} \int_{s}^{T}\left(L_{r}^{v} \bar{V}(r, x), \varphi(r, x)\right) d r d x \\
\geq & \int_{R^{d}}(g(x), \varphi(T, x)) d x+\int_{R^{d}} \int_{s}^{T}\left(h\left(r, x, v_{r}\right), \varphi(r, x)\right) d r d x \\
& +\int_{R^{d}} \int_{s}^{T}\left(\mathcal{L}_{r}^{v} \bar{V}(r, x), \varphi(r, x)\right) d r d x .
\end{aligned}
$$

So

$$
\begin{aligned}
& \int_{R^{d}}(\bar{V}(s, x), \varphi(s, x)) d x \\
\geq & \int_{R^{d}}(g(x), \varphi(T, x)) d x+\int_{R^{d}} \int_{s}^{T}\left(h\left(r, x, v_{r}\right), \varphi(r, x)\right) d r d x \\
& -\int_{R^{d}} \int_{s}^{T}\left(\sigma^{*} \nabla \bar{V}\right)(r, x) \varphi(r, x) d W_{r} d x .
\end{aligned}
$$


By Definition 3.1 we can also get, for any small $\varepsilon>0$, there exists a control $v^{\prime} \in \mathcal{U}$, we have

$$
\begin{aligned}
& \int_{R^{d}} \int_{s}^{T}\left(\bar{V}(r, x), \partial_{r} \varphi(r, x)\right) d r d x+\int_{R^{d}}(\bar{V}(s, x), \varphi(s, x)) d x-\varepsilon \\
\leq & \int_{R^{d}}(g(x), \varphi(T, x)) d x+\int_{R^{d}} \int_{s}^{T}\left(h\left(r, x, v_{r}\right), \varphi(r, x)\right) d r d x \\
& +\int_{R^{d}} \int_{s}^{T}\left(\mathcal{L}_{r}^{v^{\prime}} \bar{V}(r, x), \varphi(r, x)\right) d r d x .
\end{aligned}
$$

Taking (40) into (42), we can get

$$
\begin{aligned}
& \int_{R^{d}}(\bar{V}(s, x), \varphi(s, x)) d x-\varepsilon \\
\leq & \int_{R^{d}}(g(x), \varphi(T, x)) d x+\int_{R^{d}} \int_{s}^{T}\left(h\left(r, x, v_{r}^{\prime}\right), \varphi(r, x)\right) d r d x \\
& -\int_{R^{d}} \int_{s}^{T}\left(\sigma^{*} \nabla \bar{V}\right)(r, x) \varphi(r, x) d W_{r} d x .
\end{aligned}
$$

Let us make the change of variable $y=\widehat{x}_{r}^{t, x, v}$ in each term of (41), then

$$
\begin{aligned}
\int_{R^{d}}(\bar{V}(s, x), \varphi(s, x)) d x & =\int_{R^{d}}\left(\bar{V}\left(s, x_{s}^{t, y, v}\right), \varphi(y)\right) d y \\
\int_{R^{d}}(g(x), \varphi(T, x)) d x & =\int_{R^{d}}\left(g\left(x_{T}^{t, y, v}\right), \varphi(y)\right) d y \\
\int_{R^{d}} \int_{s}^{T}\left(h\left(r, x, v_{r}\right), \varphi(r, x)\right) d r d x & =\int_{R^{d}} \int_{s}^{T}\left(h\left(r, x_{r}^{t, y, v}, v_{r}\right), \varphi(y)\right) d r d y \\
\int_{R^{d}} \int_{s}^{T}\left(\left(\sigma^{*} \nabla \bar{V}\right)(r, x), \varphi(r, x)\right) d W_{r} d x & =\int_{R^{d}} \int_{s}^{T}\left(\left(\sigma^{*} \nabla \bar{V}\right)\left(r, x_{r}^{t, y, v}\right), \varphi(y)\right) d W_{r} d y .
\end{aligned}
$$

So (41) becomes

$$
\begin{aligned}
& \int_{R^{d}} \bar{V}\left(s, x_{s}^{t, y, v}\right) \varphi(y) d y \\
\geq & \int_{R^{d}} g\left(x_{T}^{t, y, v}\right) \varphi(y) d y+\int_{s}^{T} \int_{R^{d}}\left(h\left(r, x_{r}^{t, y, v}, v_{r}\right), \varphi(y)\right) d y d r \\
& -\int_{s}^{T} \int_{R^{d}}\left(\left(\sigma^{*} \nabla \bar{V}\right)\left(r, x_{r}^{t, y, v}\right), \varphi(y)\right) d y d W_{r} .
\end{aligned}
$$

Since $\varphi$ is arbitrary, we have proven that for $\rho(y) d y$ almost every $y$,

$$
\bar{V}\left(s, x_{s}^{t, y, v}\right) \geq g\left(x_{T}^{t, y, v}\right)+\int_{s}^{T} h\left(r, x_{r}^{t, y, v}, v_{r}\right) d r-\int_{s}^{T}\left(\sigma^{*} \nabla \bar{V}\right)\left(r, x_{r}^{t, y, v}\right) d W_{r} .
$$

So

$$
\bar{V}\left(s, x_{s}^{t, y, v}\right) \geq E\left\{g\left(x_{T}^{t, y, v}\right)+\int_{s}^{T} h\left(r, x_{r}^{t, y, v}, v_{r}\right) d r \mid \mathcal{F}_{s}^{t}\right\}
$$


In particular, when $s=t$, we get

$$
\bar{V}(t, y) \geq E\left\{g\left(x_{T}^{t, y, v}\right)+\int_{t}^{T} h\left(r, x_{r}^{t, y, v}, v_{r}\right) d r\right\} .
$$

Let us make the same change of variable $y=\widehat{x}_{r}^{t, x, v^{\prime}}$ in each term of (43), so (43) becomes

$$
\begin{aligned}
& \int_{R^{d}} \bar{V}\left(s, x_{s}^{t, y, v^{\prime}}\right) \varphi(y) d y-\varepsilon \\
\leq & \int_{R^{d}} g\left(x_{T}^{t, y, v^{\prime}}\right) \varphi(y) d y+\int_{s}^{T} \int_{R^{d}}\left(h\left(r, x_{r}^{t, y, v^{\prime}}, v_{r}^{\prime}\right), \varphi(y)\right) d y d r \\
& -\int_{s}^{T} \int_{R^{d}}\left(\sigma^{*} \nabla \bar{V}\right)\left(r, x_{r}^{t, y, v^{\prime}}\right) \varphi(y) d y d W_{r} .
\end{aligned}
$$

Since $\varphi$ is arbitrary, we have proven that for $\rho(y) d y$

$$
\begin{gathered}
\bar{V}\left(s, x_{s}^{t, y, v^{\prime}}\right)-\frac{\varepsilon}{\int_{R^{d}} \varphi(y) d y} \leq \quad g\left(x_{T}^{t, y, v^{\prime}}\right)+\int_{s}^{T} h\left(r, x_{r}^{t, y, v^{\prime}}, v_{r}^{\prime}\right) d r \\
-\int_{s}^{T}\left(\sigma^{*} \nabla \bar{V}\right)\left(r, x_{r}^{t, y, v^{\prime}}\right) d W_{r} . \\
\bar{V}\left(s, x_{s}^{t, y, v^{\prime}}\right)-\frac{\varepsilon}{\int_{R^{d}} \varphi(y) d y} \leq E\left\{g\left(x_{T}^{t, y, v^{\prime}}\right)+\int_{s}^{T} h\left(r, x_{r}^{t, y, v^{\prime}}, v_{r}^{\prime}\right) d r \mid \mathcal{F}_{s}^{t}\right\} .
\end{gathered}
$$

In particular, when $s=t$, we can get

$$
\bar{V}(t, y)-\frac{\varepsilon}{\int_{R^{d}} \varphi(y) d y} \leq E\left\{g\left(x_{T}^{t, y, v^{\prime}}\right)+\int_{t}^{T} h\left(r, x_{r}^{t, y, v^{\prime}}, v_{r}^{\prime}\right) d r\right\} .
$$

Combining (45) with (47), we know that

$$
\bar{V}(t, y)=\sup _{v \in \mathcal{U}} E\left\{g\left(x_{T}^{t, y, v}\right)+\int_{t}^{T} h\left(r, x_{r}^{t, y, v}, v_{r}\right) d r\right\} .
$$

Then $\bar{V}(t, x)$ is also the value of $\sup _{v \in \mathcal{U}} J(t, x, v)$, from uniqueness of the solution of cost functional and the uniqueness of supremum, we get the uniqueness of the weak solution for PDEs $(4)$, and $\bar{V}(t, x)=V(t, x)$.

\section{Sobolev weak solution for the HJB equation corresponding to stochastic recursive control problem}

In this section we consider the Sobolev weak solution for HJB equation related to stochastic recursive optimal control problem

The control system is defined by (1), and the cost function is defined by the following BSDE:

$$
Y_{s}^{t, \zeta ; v}=g\left(x_{T}^{t, \zeta ; v}\right)+\int_{s}^{T} f\left(r, x_{r}^{t, \zeta ; v}, Y_{r}^{t, \zeta ; v}, Z_{r}^{t, \zeta ; v}, v_{r}\right) d r-\int_{s}^{T} Z_{r}^{t, \zeta ; v} d W_{r},
$$


where

$$
\begin{aligned}
& g: R^{n} \rightarrow R, \\
& f:[0, T] \times R^{n} \times R \times R^{d} \times \mathcal{U} \rightarrow R,
\end{aligned}
$$

and they satisfy the following conditions:

(H5) $g \in L^{2}\left(R^{d}, \rho(x) d x\right)$.

(H6) $f$ measurable in $(t, x, y, z, v)$ and for any $r \in[t, T]$,

$$
E \int_{0}^{T}\left|f\left(r, 0,0,0, v_{r}\right)\right|^{2} d r \leq M,
$$

functions $f$ and $g$ are continuous and controlled by $C(1+|x|+|y|+|z|+|v|)$, and satisfy Lipschitz condition in $(x, y, z)$, i.e. there exists a constant $L>0$ such that for any $x_{1}, x_{2} ; y_{1}, y_{2}$ and $z_{1}, z_{2}$

$$
\begin{aligned}
& \left|f\left(t, x_{1}, y_{1}, z_{1}, v\right)-f\left(t, x_{2}, y_{2}, z_{2}, v\right)\right|+\left|g\left(x_{1}\right)-g\left(x_{2}\right)\right| \\
\leq & L\left(\left|x_{1}-x_{2}\right|+\left|y_{1}-y_{2}\right|+\left|z_{1}-z_{2}\right|\right) .
\end{aligned}
$$

Obviously, under the above assumptions (H1)-(H2), (H5)-(H6), for a given control $v(\cdot) \in \mathcal{U}$, there exists a unique solution $\left(Y^{t, \zeta ; v}, Z^{t, \zeta ; v}\right) \in S^{2}(0, T ; R) \times H^{2}\left(0, T ; R^{d}\right)$. We introduce the associated cost functional:

$$
J(t, x ; v):=\left.Y_{s}^{t, x ; v}\right|_{s=t}, \quad(t, x) \in[0, T] \times R^{n},
$$

and define the value function of the stochastic optimal control problem

$$
u(t, x):=e s s \sup _{v \in \mathcal{U}} J(t, x ; v), \quad(t, x) \in[0, T] \times R^{n},
$$

From Peng [13],[14], Wu and $\mathrm{Yu}[16]$, we know that the celebrated dynamic programming principle still holds for this recursive stochastic optimal control problem and the following HJB equation deduced from this principle:

$$
\left\{\begin{array}{l}
\frac{\partial u}{\partial t}(t, x)+\sup _{v \in \mathcal{U}}\{\mathcal{L}(t, x, v) u(t, x)+f(t, x, u(t, x), \sigma \nabla u(t, x), v)\}=0, \\
u(T, x)=g(x),
\end{array}\right.
$$

where $\mathcal{L}$ is a family of second order linear partial differential operators,

$$
\begin{aligned}
\mathcal{L}(t, x, q) \varphi= & \frac{1}{2} \operatorname{tr}\left[\sigma\left(t, x, v_{t}\right) \sigma\left(t, x, v_{t}\right)^{T} D^{2} \varphi\right] \\
& +\left\langle b\left(t, x, v_{t}\right), D \varphi\right\rangle .
\end{aligned}
$$

The difference between HJB (51) and (4) is that $f$ in (51) depends on $u(t, x)$ and also $\sigma \nabla u(t, x)$. However, under (H1)-(H2) and (H5)-(H6) the value function can not be differentiable about $(t, x)$, we only know its continuity, so we should study the weak solution. There are much work about viscosity solution for HJB equation (such as Peng [13], [14], Wu-Yu [16], etc). In this section, we study another kind of weak solution-Sobolev weak solution for HJB equation (51). 
Now, we first give the definition of a Sobolev solution for (51), such that

$$
\left\{\begin{array}{c}
\frac{\partial u}{\partial t}+\sup _{v \in \mathcal{U}}\left\{\frac{1}{2} \operatorname{tr}\left[\sigma \sigma^{T} \frac{\partial^{2} u}{\partial x^{2}}(t, x)\right]+\left\langle\frac{\partial u}{\partial x}, b(t, x, v)\right\rangle\right. \\
\left.+f\left(t, x, u, \sigma^{*} \nabla u, v\right)\right\}=0, \\
(t, x) \in[0, T) \times R^{n}, \\
\left.u\right|_{t=T}=g(x), \quad x \in R^{n} .
\end{array}\right.
$$

Definition 4.1. We say that $V$ is a weak solution of the equation (51), if

(i) $V \in L^{2}([0, T] ; H)$, i.e.,

$$
\int_{0}^{T}\|V(t)\|_{H}^{2} d t=\int_{0}^{T}\left(\int_{R^{d}}|V(t, x)|^{2} \rho(x) d x+\int_{R^{d}}\left|\left(\sigma^{*} \nabla V\right)(t, x)\right|^{2} \rho(x) d x\right) d t<\infty .
$$

(ii) For any nonnegative $\varphi \in C_{c}^{1, \infty}\left([0, T] \times R^{d}\right)$ and for any $v \in \mathcal{U}$, then

$$
\begin{aligned}
& \int_{R^{d}} \int_{s}^{T}\left(V(r, x), \partial_{r} \varphi(r, x)\right) d r d x+\int_{R^{d}}(V(s, x), \varphi(s, x)) d x \\
\geq & \int_{R^{d}}(g(x), \varphi(T, x)) d x+\int_{R^{d}} \int_{s}^{T}\left(f\left(r, x, V, \sigma^{*} \nabla V, v_{r}\right), \varphi(r, x)\right) d r d x \\
& +\int_{R^{d}} \int_{s}^{T}\left(\mathcal{L}_{r}^{v} V(r, x), \varphi(r, x)\right) d r d x,
\end{aligned}
$$

where $\left(L_{r} V(r, x), \varphi(r, x)\right)=\int_{R^{d}}\left(\frac{1}{2}(\nabla V \sigma)\left(\sigma^{*} \nabla \varphi\right)+V \operatorname{div}(b-A) \varphi\right) d x$ with $A_{i}=\frac{1}{2} \sum_{k=1}^{d} \frac{\partial a_{k, i}}{\partial x_{k}}$.

(iii) For any nonnegative $\varphi \in C_{c}^{1, \infty}\left([0, T] \times R^{d}\right)$ and for any small $\varepsilon>0$, there exists a control $v^{\prime} \in \mathcal{U}$, such that

$$
\begin{aligned}
& \int_{R^{d}} \int_{s}^{T}\left(V(r, x), \partial_{r} \varphi(r, x)\right) d r d x+\int_{R^{d}}(V(s, x), \varphi(s, x)) d x-\varepsilon \\
\leq & \int_{R^{d}}(g(x), \varphi(T, x)) d x+\int_{R^{d}} \int_{s}^{T}\left(f\left(r, x, V, \sigma^{*} \nabla V, v_{r}^{\prime}\right), \varphi(r, x)\right) d r d x \\
& +\int_{R^{d}} \int_{s}^{T}\left(\mathcal{L}_{r}^{v^{\prime}} V(r, x), \varphi(r, x)\right) d r d x
\end{aligned}
$$

Before we begin to give the main result of this section, we first introduce a definition in BSDE theory which comes from Peng [15].

Definition 4.2. If $\left(Y_{t}\right)_{0 \leq t \leq T}$ is the solution of the $\operatorname{BSDE}(48)$ on $[0, T]$, then $Y_{t}$ is called a g-solution and also a g-martingale. An $\mathcal{F}_{t}$-progressively measurable real-value process $\left(Y_{t}\right)_{0 \leq t \leq T}$ is called a g-supermartingale (resp. g-submartingale) on $[0, T]$ if for each (deterministic time) $t \leq T, E\left|Y_{t}\right|^{2}<\infty$, and the g-solution $y_{t}$ on $[0, t]$ with terminal condition $y_{t}=Y_{t}$ satisfies $y_{s} \leq Y_{s}$ (resp. $y_{s} \geq Y_{s}$ ) for all deterministic time $s \leq t$. The process $Y_{t}$ is called a g-supersolution if $Y_{t}$ is a solution of the following form BSDE:

$$
Y_{t}=\xi+\int_{t}^{T} f\left(s, Y_{s}, Z_{s}\right) d s+\left(A_{T}-A_{t}\right)-\int_{t}^{T} Z_{s} d W_{s}, \quad 0 \leq t \leq T,
$$

where $A_{t}$ is a given RCLL increasing process with $A_{0}=0$ and $E\left(A_{T}\right)^{2}<\infty$.

And then, we have 
Lemma 4.1 We assume (H1)-(H2) and (H5)-(H6), for any $v$, we know that $V\left(s, x_{s}^{t, x, v}\right)$ satisfying

$$
V\left(s, x_{s}^{t, x, v}\right) \geq Y_{s}^{s, x_{s}^{t, x, v}, v}
$$

and $E\left|V\left(s, x_{s}^{t, x, v}\right)\right|^{2}<\infty$, then $V\left(s, x_{s}^{t, x, v}\right)$ is a g-supermartingale. Moreover there exists a unique increasing process $\left(A_{r}\right)$ with $A_{t}=0$ and $E\left[\left(A_{T}\right)^{2}\right]<\infty$ such that $V\left(s, x_{s}^{t, x, v}\right)$ coincides with the unique solution $y_{s}^{t, x, v}$ of the BSDE:

$$
\begin{aligned}
y_{t}^{t, x, v}= & V\left(T, x_{T}^{t, x, v}\right)+\int_{t}^{T} f\left(r, x_{r}^{t, x, v}, y_{r}^{t, x, v}, Z_{r}^{t, x, v}, v_{r}\right) d r+A_{T}^{t, x, v}-A_{t}^{t, x, v} \\
& -\int_{t}^{T} Z_{r}^{t, x, v} d W_{r}
\end{aligned}
$$

where $Z_{r}^{t, x, v}=\sigma^{*} \nabla V\left(r, x_{r}^{t, x, v}\right)$ in the sense of Definition 4.1.

Proof: This lemma is similar to the Theorem 3.3 in Peng [15], but our problem is related to the value function satisfying dynamic programming principle, our proof idea comes from Peng [15].

We first consider the following family of BSDE parameterized by $i=1,2, \cdots$

$$
\begin{aligned}
y_{t}^{t, x, v ; i}= & V\left(T, x_{T}^{t, x, v}\right)+\int_{t}^{T} f\left(r, x_{r}^{t, x, v}, y_{r}^{t, x, v ; i}, Z_{r}^{t, x, v ; i}, v_{r}\right) d r \\
& +i \int_{t}^{T}\left(V\left(r, x_{r}^{t, x, v}\right)-y_{r}^{t, x, v ; i}\right) d r-\int_{t}^{T} Z_{r}^{t, x, v ; i} d W_{r} .
\end{aligned}
$$

By Lemma 3.4 in [15], we know that $y_{r}^{t, x, v ; i}$ is bounded from above by $V\left(r, x_{r}^{t, x, v}\right)$. Thus $y_{r}^{t, x, v ; i}$ is a g-supersolution on $[\mathrm{t}, \mathrm{T}]$.

We know that $V\left(r, x_{r}^{t, x, v}\right)-y_{r}^{t, x, v ; i} \geq 0$, it follows from the comparison theorem of the BSDE, that $y_{r}^{t, x, v ; i} \leq y_{r}^{t, x, v ; i+1}$. Thus $\left\{y^{t, x, v ; i}\right\}$ is a sequence of continuous g-supermartingale that is monotonically converges up to a process $y_{r}^{t, x, v}$, moreover $y_{r}^{t, x, v}$ is bounded from above $V\left(r, x_{r}^{t, x, v}\right)$, by Theorem 2.4 in [15] we know that $y_{r}^{t, x, v}$ is a g-supersolution in $[t, T]$ of the following form.

$$
y_{t}^{t, x, v}=V\left(T, x_{T}^{t, x, v}\right)+\int_{t}^{T} f\left(r, x_{r}^{t, x, v}, y_{r}^{t, x, v}, Z_{r}^{t, x, v}, v_{r}\right) d r+\left(A_{T}-A_{t}\right)-\int_{t}^{T} Z_{r}^{t, x, v} d W_{r},
$$

where $A_{t}$ is a RCLL increasing process. It then remains to prove that $y_{r}^{t, x, v}=V\left(r, x_{r}^{t, x, v}\right)$.

We denote

$$
A_{r}^{t, x, v ; i}:=i \int_{t}^{r}\left(V\left(s, x_{s}^{t, x, v}\right)-y_{s}^{t, x, v ; i}\right) d s .
$$


From BSDE (56), we have

$$
\begin{aligned}
A_{T}^{t, x, v ; i}-A_{t}^{t, x, v ; i}= & y_{t}^{t, x, v ; i}-V\left(T, x_{T}^{t, x, v}\right)-\int_{t}^{T} f\left(r, x_{r}^{t, x, v}, v_{r}\right) d r+\int_{t}^{T} Z_{r}^{t, x, v ; i} d W_{r} \\
\leq & \left|y_{t}^{t, x, v ; i}\right|+\left|V\left(T, x_{T}^{t, x, v}\right)\right|+\int_{t}^{T} f\left(r, 0,0,0, v_{r}\right) d r \\
& +\int_{t}^{T}\left(K\left|x_{r}^{t, x, v}\right|+K\left|y_{r}^{t, x, v ; i}\right|+K\left|Z_{r}^{t, x, v ; i}\right|\right) d r+\left|\int_{t}^{T} Z_{r}^{t, x, v, i} d W_{r}\right| \\
\leq & |V(t, x)|+\left|V\left(T, x_{T}^{t, x, v}\right)\right|+\int_{t}^{T} f\left(r, 0,0,0, v_{r}\right) d r \\
& +\int_{t}^{T}\left(K\left|x_{r}^{t, x, v}\right|+K\left|y_{r}^{t, x, v ; 1}\right|+K\left|V\left(r, x_{r}^{t, x, v}\right)\right|+K\left|Z_{r}^{t, x, v, i}\right|\right) d r \\
& +\left|\int_{t}^{T} Z_{r}^{t, x, v, i} d W_{r}\right|,
\end{aligned}
$$

because for any $v_{r}, E \int_{t}^{T}\left|f\left(r, 0,0,0, v_{r}\right)\right|^{2} d r \leq M$. We observe that $y_{r}^{t, x, v ; i}$ is dominated by $\left|y_{r}^{t, x, v ; 1}\right|+\left|V\left(r, x_{r}^{t, x, v}\right)\right|$. From (56), we have

$$
\begin{aligned}
& E\left|A_{T}^{t, x, v ; i}\right|^{2} \\
\leq & 8|V(t, x)|^{2}+8 E\left|g\left(x_{T}^{t, x, v}\right)\right|^{2}+8 E \int_{t}^{T}\left|f\left(r, 0,0,0, v_{r}\right)\right|^{2} d r \\
& +8 E \int_{t}^{T}\left(K^{2}\left|x_{r}^{t, x, v}\right|^{2}+K^{2}\left|y_{r}^{t, x, v ; 1}\right|^{2}+K^{2}\left|V\left(r, x_{r}^{t, x, v}\right)\right|^{2}+K^{2}\left|Z_{r}^{t, x, v, i}\right|^{2}\right) d r \\
& +8 E \int_{t}^{T}\left|Z_{r}^{t, x, v, i}\right|^{2} d r,
\end{aligned}
$$

Thus we can define a $C_{3}(t, T, x, v)$, independent of $i$, such that

$$
E\left|A_{T}^{t, x, v ; i}\right|^{2} \leq C_{3}(t, T, x, v)+8\left(K^{2}+1\right) E \int_{t}^{T}\left|Z_{r}^{t, x, v ; i}\right|^{2} d r .
$$

On the other hand, we use It $\widehat{o}$ 's formula to $\left|y_{r}^{t, x, v ; i}\right|^{2}$

$$
\begin{aligned}
& \left|y_{t}^{t, x, v ; i}\right|^{2}+E \int_{t}^{T}\left|Z_{r}^{t, x, v ; i}\right|^{2} d r \\
= & E\left|V\left(T, x_{T}^{t, x, v}\right)\right|^{2}+2 E \int_{t}^{T} y_{r}^{t, x, v ; i} f\left(r, x_{r}^{t, x, v}, y_{r}^{t, x, v ; i}, Z_{r}^{t, x, v ; i}, v_{r}\right) d r+2 E \int_{t}^{T} y_{r}^{t, x, v ; i} d A_{r}^{t, x, v ; i} \\
\leq & E\left|V\left(T, x_{T}^{t, x, v}\right)\right|^{2}+2 E \int_{t}^{T}\left(K+K^{2}\right)\left|y_{r}^{t, x, v ; i}\right|^{2} d r+\frac{1}{2} E \int_{t}^{T}\left|Z_{r}^{t, x, v ; i}\right|^{2} d r \\
& +K E \int_{t}^{T}\left|x_{r}^{t, x, v}\right|^{2} d r+E \int_{t}^{T}\left|f\left(r, 0,0,0, v_{r}\right)\right|^{2} d r+2 E\left[A_{T}^{t, x, v ; i} \sup _{t \leq s \leq T}\left|y_{s}^{t, x, v ; i}\right|\right] \\
\leq & E\left|V\left(T, x_{T}^{t, x, v}\right)\right|^{2}+4 E \int_{t}^{T}\left(K+K^{2}\right)\left[\left|y_{r}^{t, x, v ; 1}\right|^{2}+\left|V\left(r, x_{r}^{t, x, v}\right)\right|^{2}\right] d r+\frac{1}{2} E \int_{t}^{T}\left|Z_{r}^{t, x, v ; i}\right|^{2} d r \\
& +K E \int_{t}^{T}\left|x_{r}^{t, x, v}\right|^{2} d r+E \int_{t}^{T}\left|f\left(r, 0,0,0, v_{r}\right)\right|^{2} d r+\frac{1}{32\left(K^{2}+1\right)} E\left|A_{T}^{t, x, v ; i}\right|^{2} \\
& +64\left(K^{2}+1\right) E \sup _{t \leq s \leq T}\left[\left|y_{s}^{t, x, v ; 1}\right|^{2}+\left|V\left(s, x_{s}^{t, x, v}\right)\right|^{2}\right] .
\end{aligned}
$$


Thus we also can define a $C_{4}(t, T, x, v)$ satisfying

$$
E \int_{t}^{T}\left|Z_{r}^{t, x, v ; i}\right|^{2} d r \leq C_{4}(t, T, x, v)+\frac{1}{16\left(K^{2}+1\right)} E\left|A_{T}^{t, x, v ; i}\right|^{2}
$$

where the constants $C_{3}, C_{4}$ are independent of $i$, so we can get

$$
E\left|A_{T}^{t, x, v ; i}\right|^{2} \leq 2 C_{3}(t, T, x, v)+16\left(K^{2}+1\right) C_{4}(t, T, x, v) .
$$

And then

$$
i^{2} \int_{t}^{r}\left(V\left(s, x_{s}^{t, x, v}\right)-y_{s}^{t, x, v ; i}\right)^{2} d s \leq 2 C_{3}(t, T, x, v)+16\left(K^{2}+1\right) C_{4}(t, T, x, v) .
$$

We let $i \rightarrow \infty$, it also hold, so we can get $y_{r}^{t, x, v}=V\left(r, x_{r}^{t, x, v}\right)$.

The proof of uniqueness comes from the uniqueness proof of the g-supersolution (Proposition 1.6 in [15]). If both there have another solution $A_{r}^{\prime}$ and $Z^{\prime t, x, v}$ satisfying (57), then we apply It $\widehat{o}$ 's formula to $\left(y_{t}-y_{t}\right)^{2}(\equiv 0)$ on $[0, T]$ and take expectation:

$$
E \int_{t}^{T}\left|Z_{s}^{t, x, v}-Z_{s}^{\prime t, x, v}\right|^{2} d s+E\left[\left(A_{T}-A_{T}^{\prime}\right)-\left(A_{t}-A_{t}^{\prime}\right)\right]^{2}=0 .
$$

for any $t \in[0, T]$. Thus $Z^{t, x, v} \equiv Z^{\prime t, x, v} A_{r} \equiv A_{r}^{\prime}$.

Now we consider BSDE (56), we have $i\left(V\left(r, x_{r}^{t, x, v}\right)-y_{r}^{t, x, v ; i}\right)$ is Lipschitz about $y_{r}^{t, x, v ; i}$, and we have

$$
E \int_{t}^{T} \int_{R^{d}}\left(\left|f\left(r, x, 0,0, v_{r}\right)\right|^{2}+|V(r, x)|^{2}\right) \rho(x) d x d r<\infty
$$

and the $b, \sigma$ satisfying the (H2), so by Lemma 4.1 in [11], we know $Z_{r}^{t, x, v ; i}=\sigma^{*} \nabla y_{r}^{t, x, v ; i}$.

By the estimate of the BSDE given below, we know that there exists a $Z_{r}^{t, x, v}$ which is the weak limit of $Z_{r}^{t, x, v ; i}$ in $L^{2}\left([t, T], R^{d}\right)$,

$$
\begin{aligned}
& E\left|Y_{t}^{t, x, v, m}-Y_{t}^{t, x, v, n}\right|^{2}+E \int_{t}^{T}\left|Z_{r}^{t, x, v, m}-Z_{r}^{t, x, v, n}\right|^{2} d r \\
\leq & E \int_{t}^{T}\left(C+C^{2}\right)\left|Y_{r}^{t, x, v, m}-Y_{r}^{t, x, v, n}\right|^{2} d r+\frac{1}{4} E \int_{t}^{T}\left|Z_{r}^{t, x, v, m}-Z_{r}^{t, x, v, n}\right|^{2} d r \\
& +E \int_{t}^{T}\left|Y_{r}^{t, x, v, m}-Y_{r}^{t, x, v, n}\right| d\left(A_{r}^{t, x, v, m}-A_{r}^{t, x, v, n}\right) \\
\leq & E \int_{t}^{T}\left(C+C^{2}\right)\left|Y_{r}^{t, x, v, m}-Y_{r}^{t, x, v, n}\right|^{2} d r+\frac{1}{4} E \int_{t}^{T}\left|Z_{r}^{t, x, v, m}-Z_{r}^{t, x, v, n}\right|^{2} d r \\
& +E \sup _{t \leq r \leq T}\left|Y_{r}^{t, x, v, m}-Y_{r}^{t, x, v, n}\right|\left(A_{T}^{t, x, v, m}-A_{T}^{t, x, v, n}\right) \\
\leq & E \int_{t}^{T}\left(C+C^{2}\right)\left|Y_{r}^{t, x, v, m}-Y_{r}^{t, x, v, n}\right|^{2} d r+\frac{1}{4} E \int_{t}^{T}\left|Z_{r}^{t, x, v, m}-Z_{r}^{t, x, v, n}\right|^{2} d r \\
& +\left(E \sup _{t \leq r \leq T}\left|Y_{r}^{t, x, v, m}-Y_{r}^{t, x, v, n}\right|^{2}\right)^{\frac{1}{4}}\left(E\left(A_{T}^{t, x, v, m}-A_{T}^{t, x, v, n}\right)^{2}\right)^{\frac{1}{2}} .
\end{aligned}
$$


So

$$
\begin{aligned}
& E \int_{t}^{T}\left|Z_{r}^{t, x, v, m}-Z_{r}^{t, x, v, n}\right|^{2} d r \\
\leq & C_{1} E \int_{t}^{T}\left|Y_{r}^{t, x, v, m}-Y_{r}^{t, x, v, n}\right|^{2} d r \\
& +\left(E \sup _{t \leq r \leq T}\left|Y_{r}^{t, x, v, m}-Y_{r}^{t, x, v, n}\right|^{2}\right)^{\frac{1}{4}}\left(E\left(A_{T}^{t, x, v, m}-A_{T}^{t, x, v, n}\right)^{2}\right)^{\frac{1}{2}} .
\end{aligned}
$$

Because $E\left(A_{T}^{t, x, v ; i}\right)^{2}$ is bounded, and $Y_{r}^{t, x, v ; m}$ is convergent, so

$$
\lim _{m, n \rightarrow \infty} E \int_{t}^{T}\left|Z_{r}^{t, x, v ; n}-Z_{r}^{t, x, v ; m}\right|^{2} d r=0
$$

then $Z_{r}^{t, x, v}=\sigma^{*} \nabla y_{r}^{t, x, v}=\sigma^{*} \nabla V\left(r, x_{r}^{t, x, v}\right)$.

On the other hand, we have

Lemma 4.2 We assume (H1)-(H2) and (H5)-(H6), for any small $\varepsilon>0$, there exists a control $v^{\prime} \in \mathcal{U}, V\left(s, x_{s}^{t, x, v^{\prime}}\right)$ satisfying (52) and

$$
V\left(s, x_{s}^{t, x, v^{\prime}}\right) \leq Y_{s}^{s, x_{s}^{t, x, v^{\prime}}, v^{\prime}}+\varepsilon
$$

then $V\left(s, x_{s}^{t, x, v^{\prime}}\right)$ is a g-submartingale. By the nonlinear Doob-Meyer decomposition theorem, there exists a unique increasing process $\left(A_{r}^{\prime}\right)$ with $A_{t}^{\prime}=0$ and $E\left[\left(A_{T}^{\prime}\right)^{2}\right]<\infty$ such that $V\left(s, x_{s}^{t, x, v^{\prime}}\right)$ coincides with the unique solution $y_{s}^{t, x, v}$ of the BSDE:

$$
\begin{aligned}
y_{t}^{t, x, v^{\prime}}= & V\left(T, x_{T}^{t, x, v^{\prime}}\right)+\varepsilon+\int_{t}^{T} f\left(r, x_{r}^{t, x, v^{\prime}}, y_{r}^{t, x, v^{\prime}}, Z_{r}^{t, x, v^{\prime}}, v_{r}^{\prime}\right) d r \\
& -\left(A_{T}^{t, x, v^{\prime}}-A_{t}^{t, x, v^{\prime}}\right)-\int_{t}^{T} Z_{r}^{t, x, v^{\prime}} d W_{r} .
\end{aligned}
$$

where $Z_{r}^{t, x, v^{\prime}}=\sigma^{*} \nabla V\left(r, x_{r}^{t, x, v^{\prime}}\right)$ in the sense of Definition 4.1 .

The proof of Lemma 4.2 is similar to Lemma 4.1, hence we omit it.

Now, we give the main result of this section.

Theorem 4.1 Under the assumption (H1)-(H2) and (H5)-(H6), the value function $V(t, x)$ defined in (50) is the unique Sobolev solution of the PDE (51).

Proof: Existence: In the stochastic recursive optimal control problem, the value function $V(t, x)$ defined by (50) satisfies the Bellman's dynamic programming principle. By Lemma 4.1 and lemma 4.2, we know that, for any $v \in \mathcal{U}$, there have a unique increasing process $A_{s}^{t, x, v}, V\left(s, x_{s}^{t, x, v}\right)$ satisfy the following BSDE:

$$
\begin{aligned}
V\left(s, x_{s}^{t, x, v}\right)= & V\left(T, x_{T}^{t, x, v}\right)+\int_{s}^{T} f\left(r, x_{r}^{t, x, v}, V\left(r, x_{r}^{t, x, v}\right), \sigma^{*} \nabla V\left(r, x_{r}^{t, x, v}\right), v_{r}\right) d r \\
& +\left(A_{T}^{t, x, v}-A_{s}^{t, x, v}\right)-\int_{s}^{T} \sigma^{*} \nabla V\left(r, x_{r}^{t, x, v}\right) d W_{r}
\end{aligned}
$$


So we can get

$$
\begin{aligned}
V\left(s, x_{s}^{t, x, v}\right) \geq & V\left(T, x_{T}^{t, x, v}\right)+\int_{s}^{T} f\left(r, x_{r}^{t, x, v}, V\left(r, x_{r}^{t, x, v}\right), \sigma^{*} \nabla V\left(r, x_{r}^{t, x, v}\right), v_{r}\right) d r \\
& -\int_{s}^{T} \sigma^{*} \nabla V\left(r, x_{r}^{t, x, v}\right) d W_{r} .
\end{aligned}
$$

For any small $\varepsilon>0$ there exists a control $v^{\prime} \in \mathcal{U}, V\left(s, x_{s}^{t, x, v^{\prime}}\right)$ satisfies the following BSDE:

$$
\begin{aligned}
V\left(s, x_{s}^{t, x, v^{\prime}}\right)-\varepsilon= & V\left(T, x_{T}^{t, x, v^{\prime}}\right)+\int_{s}^{T} f\left(r, x_{r}^{t, x, v^{\prime}}, V\left(r, x_{r}^{t, x, v^{\prime}}\right), \sigma^{*} \nabla V\left(r, x_{r}^{t, x, v^{\prime}}\right), v_{r}^{\prime}\right) d r \\
& -\left(A_{T}^{t, x, v^{\prime}}-A_{s}^{t, x, v^{\prime}}\right)-\int_{s}^{T} \sigma^{*} \nabla V\left(r, x_{r}^{t, x, v^{\prime}}\right) d W_{r} .
\end{aligned}
$$

So we can get

$$
\begin{aligned}
V\left(s, x_{s}^{t, x, v^{\prime}}\right)-\varepsilon \leq & V\left(T, x_{T}^{t, x, v^{\prime}}\right)+\int_{s}^{T} f\left(r, x_{r}^{t, x, v^{\prime}}, V\left(r, x_{r}^{t, x, v^{\prime}}\right), \sigma^{*} \nabla V\left(r, x_{r}^{t, x, v^{\prime}}\right), v_{r}^{\prime}\right) d r \\
& -\int_{s}^{T} \sigma^{*} \nabla V\left(r, x_{r}^{t, x, v^{\prime}}\right) d W_{r} .
\end{aligned}
$$

We can deduce by the equivalence of norm result (Lemma 3.2) that $V \in L^{2}([t, T], H)$. Indeed, in the stochastic recursive optimal control problem, the cost function can be regarded as a solution of BSDE:

$$
Y_{s}^{t, x, v}=g\left(x_{T}^{t, x, v}\right)+\int_{s}^{T} f\left(r, x_{r}^{t, x, v}, Y_{r}^{t, x, v}, Z_{r}^{t, x, v}, v_{r}\right) d r-\int_{s}^{T} Z_{r}^{t, x, v} d W_{r},
$$

by the estimate of the BSDE, and the (H1)-(H2) and (H5)-(H6) we know that

$$
\begin{aligned}
& \int_{R^{d}} E\left(\left|Y_{t}^{t, x, v}\right|^{2}+\int_{t}^{T}\left|Z_{r}^{t, x, v}\right|^{2} d r\right) \rho(x) d x \\
\leq & K \int_{R^{d}} E\left|g\left(x_{T}^{t, x, v}\right)\right|^{2} \rho(x) d x+K \int_{R^{d}} \int_{t}^{T} E\left|f\left(r, x_{r}^{t, x, v}, 0,0, v_{r}\right)\right|^{2} d r \rho(x) d x \\
\leq & K C \int_{R^{d}}|g(x)|^{2} \rho(x) d x+K C \int_{R^{d}} \int_{t}^{T}\left|f\left(r, x, 0,0, v_{r}\right)\right|^{2} d r \rho(x) d x<\infty \\
\leq & K C \int_{R^{d}}|g(x)|^{2} \rho(x) d x+K C \int_{R^{d}} \int_{t}^{T} C_{1} d r \rho(x) d x<\infty \\
= & K C \int_{R^{d}}|g(x)|^{2} \rho(x) d x+(T-t) C_{1} C K<\infty,
\end{aligned}
$$

So for any $v, Y_{r}^{t, x, v} \in H$, here $H$ is a Hilbert space, $V(t, x)=\sup _{v \in U} Y_{t}^{t, x, v}$. From $U$ is compact set of $R^{k}$ and Lemma 6.2 in [14], we know that $V(t, \cdot)$ is also in $H$. 
Next because (68) holds, then for any nonnegative $\varphi \in C_{c}^{\infty}\left(R^{d}\right)$, we have

$$
\begin{aligned}
& \int_{R^{d}}\left(V\left(s, x_{s}^{t, x, v}\right), \varphi(x)\right) d x \\
\geq & \int_{R^{d}}\left(V\left(T, x_{T}^{t, x, v}\right), \varphi(x)\right) d x \\
& +\int_{R^{d}} \int_{s}^{T}\left(f\left(r, x_{r}^{t, x, v}, V\left(r, x_{r}^{t, x, v}\right), \sigma^{*} \nabla V\left(r, x_{r}^{t, x, v}\right), v_{r}\right), \varphi(x)\right) d r d x \\
& -\int_{R^{d}} \int_{s}^{T}\left(\sigma^{*} \nabla V\left(r, x_{r}^{t, x, v}\right), \varphi(x)\right) d W_{r} d x .
\end{aligned}
$$

That is

$$
\begin{aligned}
& \int_{R^{d}}(V(s, x), \varphi(s, x)) d x \\
\geq & \int_{R^{d}}(g(x), \varphi(T, x)) d x+\int_{R^{d}} \int_{s}^{T}\left(f\left(r, x, V(r, x), \sigma^{*} \nabla V(r, x), v_{r}\right), \varphi(r, x)\right) d r d x \\
& -\int_{R^{d}} \int_{s}^{T}\left(\sigma^{*} \nabla V(r, x), \varphi(r, x)\right) d W_{r} d x .
\end{aligned}
$$

Furthermore, using Lemma 3.1, we have

$$
\begin{aligned}
& -\int_{R^{d}} \int_{s}^{T} \sigma^{*} \nabla V(r, x) \varphi(r, x) d W_{r} d x \\
= & -\int_{R^{d}} \sum_{j=1}^{d} \int_{s}^{T}\left(\sum_{i=1}^{d} \sigma_{i, j}(r, x) \frac{\partial V}{\partial x_{i}}(r, x), \varphi(r, x)\right) d W_{r}^{j} \\
= & \int_{R^{d}} \int_{s}^{T}\left(\mathcal{L}^{v} V(r, x), \varphi(r, x)\right) d r-\int_{R^{d}} \int_{s}^{T}\left(V(r, x), \partial_{r} \varphi(r, x)\right) d r d x .
\end{aligned}
$$

Taking (75) into (74), so we can get

$$
\begin{aligned}
& \int_{R^{d}} \int_{s}^{T}\left(V(r, x), \partial_{r} \varphi(r, x)\right) d r d x+\int_{R^{d}}(V(s, x), \varphi(s, x)) d x \\
\geq & \int_{R^{d}}(g(x), \varphi(T, x)) d x+\int_{R^{d}} \int_{s}^{T}\left(f\left(r, x, V(r, x), \sigma^{*} \nabla V(r, x), v_{r}\right), \varphi(r, x)\right) d r d x \\
& +\int_{R^{d}} \int_{s}^{T}\left(\mathcal{L}_{r}^{v} V(r, x), \varphi(r, x)\right) d r d x .
\end{aligned}
$$

By virtue of the same techniques and methods, because (70) holds, then for any nonnegative $\varphi \in C_{c}^{\infty}\left(R^{d}\right)$, we take $\varepsilon=\frac{\varepsilon^{\prime}}{\int_{R^{d}} \varphi(x) d x}$, so

$$
\begin{aligned}
& \int_{R^{d}}\left(V\left(s, x_{s}^{t, x, v^{\prime}}\right), \varphi(x)\right) d x-\varepsilon^{\prime} \\
\leq & \int_{R^{d}}\left(g\left(x_{T}^{t, x, v^{\prime}}\right), \varphi(x)\right) d x \\
& +\int_{R^{d}} \int_{s}^{T}\left(f\left(r, x_{r}^{t, x, v^{\prime}}, V\left(r, x_{r}^{t, x, v^{\prime}}\right), \sigma^{*} \nabla V\left(r, x_{r}^{t, x, v^{\prime}}\right), v_{r}^{\prime}\right), \varphi(x)\right) d r d x \\
& -\int_{R^{d}} \int_{s}^{T}\left(\sigma^{*} \nabla V\left(r, x_{r}^{t, x, v^{\prime}}\right), \varphi(x)\right) d W_{r} d x,
\end{aligned}
$$


that is

$$
\begin{aligned}
& \int_{R^{d}}(V(s, x), \varphi(s, x)) d x-\varepsilon^{\prime} \\
\leq & \int_{R^{d}}(g(x), \varphi(T, x)) d x+\int_{R^{d}} \int_{s}^{T}\left(f\left(r, x, V(r, x), \sigma^{*} \nabla V(r, x), v_{r}^{\prime}\right), \varphi(r, x)\right) d r d x \\
& -\int_{R^{d}} \int_{s}^{T}\left(\sigma^{*} \nabla V(r, x), \varphi(r, x)\right) d W_{r} d x .
\end{aligned}
$$

Taking (75) into (78), we can get

$$
\begin{aligned}
& \int_{R^{d}} \int_{s}^{T}\left(V(r, x), \partial_{r} \varphi(r, x)\right) d r d x+\int_{R^{d}}(V(s, x), \varphi(s, x)) d x-\varepsilon^{\prime} \\
\leq & \int_{R^{d}}(g(x), \varphi(T, x)) d x+\int_{R^{d}} \int_{s}^{T}\left(f\left(r, x, V(r, x), \sigma^{*} \nabla V(r, x), v_{r}^{\prime}\right), \varphi(r, x)\right) d r d x \\
& +\int_{R^{d}} \int_{s}^{T}\left(\mathcal{L}_{r}^{v^{\prime}} V(r, x), \varphi(r, x)\right) d r d x .
\end{aligned}
$$

Uniqueness:

Let $\bar{V}$ be another solution of the PDE (51). By Definition 4.1, one gets: for any $v \in \mathcal{U}$,

$$
\begin{aligned}
& \int_{R^{d}} \int_{s}^{T}\left(\bar{V}(r, x), \partial_{r} \varphi(r, x)\right) d r d x+\int_{R^{d}}(\bar{V}(s, x), \varphi(s, x)) d x \\
\geq & \int_{R^{d}}(g(x), \varphi(T, x)) d x+\int_{R^{d}} \int_{s}^{T}\left(f\left(r, x, \bar{V}(r, x), \sigma^{*} \nabla \bar{V}(r, x), v_{r}\right), \varphi(r, x)\right) d r d x \\
& +\int_{R^{d}} \int_{s}^{T}\left(\mathcal{L}_{t}^{v} \bar{V}(r, x), \varphi(r, x)\right) d r d x .
\end{aligned}
$$

By Lemma 4.5 in [11], we have

$$
\begin{aligned}
& \int_{R^{d}} \int_{s}^{T}\left(\bar{V}(r, x), \partial_{r} \varphi(r, x)\right) d r d x \\
= & \int_{R^{d}} \Sigma_{j=1}^{d} \int_{s}^{T}\left(\Sigma_{i=1}^{d}\left(\sigma_{i, j} \frac{\partial \bar{V}}{\partial x_{i}}(r, x), \varphi(r, x)\right)\right) d W_{r} d x+\int_{R^{d}} \int_{s}^{T}\left(L_{r}^{v} \bar{V}(r, x), \varphi(r, x)\right) d r d x \\
= & \int_{R^{d}} \int_{s}^{T}\left(\left(\sigma^{*} \nabla \bar{V}\right)(r, x), \varphi(r, x)\right) d W_{r} d x+\int_{R^{d}} \int_{s}^{T}\left(L_{r}^{v} \bar{V}(r, x), \varphi(r, x)\right) d r d x .
\end{aligned}
$$

Taking (81) into (80), we can get

$$
\begin{aligned}
& \int_{R^{d}}(\bar{V}(s, x), \varphi(s, x)) d x+\int_{R^{d}} \int_{s}^{T}\left(\sigma^{*} \nabla \bar{V}\right)(r, x) \varphi(r, x) d W_{r} d x \\
& +\int_{R^{d}} \int_{s}^{T}\left(L_{r}^{v} \bar{V}(r, x), \varphi(r, x)\right) d r d x \\
\geq & \int_{R^{d}}(g(x), \varphi(T, x)) d x+\int_{R^{d}} \int_{s}^{T}\left(f\left(r, x, \bar{V}(r, x), \sigma^{*} \nabla \bar{V}(r, x), v_{r}\right), \varphi(r, x)\right) d r d x \\
& +\int_{R^{d}} \int_{s}^{T}\left(\mathcal{L}_{t}^{v} \bar{V}(r, x), \varphi(r, x)\right) d r d x .
\end{aligned}
$$


So

$$
\begin{aligned}
& \int_{R^{d}}(\bar{V}(s, x), \varphi(s, x)) d x \\
\geq & \int_{R^{d}}(g(x), \varphi(T, x)) d x+\int_{R^{d}} \int_{s}^{T}\left(f\left(r, x, \bar{V}(r, x), \sigma^{*} \nabla \bar{V}(r, x), v_{r}\right), \varphi(r, x)\right) d r d x \\
& -\int_{R^{d}} \int_{s}^{T}\left(\sigma^{*} \nabla \bar{V}\right)(r, x) \varphi(r, x) d W_{r} d x .
\end{aligned}
$$

By Definition 4.1 we can also gets, for any small $\varepsilon>0$, there exists a control $v^{\prime} \in \mathcal{U}$, we have

$$
\begin{aligned}
& \int_{R^{d}} \int_{s}^{T}\left(\bar{V}(r, x), \partial_{r} \varphi(r, x)\right) d r d x+\int_{R^{d}}(\bar{V}(s, x), \varphi(s, x)) d x-\varepsilon \\
\leq & \int_{R^{d}}(g(x), \varphi(T, x)) d x+\int_{R^{d}} \int_{s}^{T}\left(f\left(r, x, \bar{V}(r, x), \sigma^{*} \nabla \bar{V}(r, x), v_{r}^{\prime}\right), \varphi(r, x)\right) d r d x \\
& +\int_{R^{d}} \int_{s}^{T}\left(\mathcal{L}_{r}^{v^{\prime}} \bar{V}(r, x), \varphi(r, x)\right) d r d x
\end{aligned}
$$

Taking (81) into (83), we can get

$$
\begin{aligned}
& \int_{R^{d}}(\bar{V}(s, x), \varphi(s, x)) d x-\varepsilon \\
\leq & \int_{R^{d}}(g(x), \varphi(T, x)) d x+\int_{R^{d}} \int_{s}^{T}\left(f\left(r, x, \bar{V}(r, x), \sigma^{*} \nabla \bar{V}(r, x), v_{r}^{\prime}\right), \varphi(r, x)\right) d r d x \\
& -\int_{R^{d}} \int_{s}^{T}\left(\sigma^{*} \nabla \bar{V}\right)(r, x) \varphi(r, x) d W_{r} d x .
\end{aligned}
$$

Let us make the change of variable $y=\widehat{x}_{r}^{t, x, v}$ in each term of (82), then

$$
\begin{gathered}
\int_{R^{d}}(\bar{V}(s, x), \varphi(s, x)) d x=\int_{R^{d}}\left(\bar{V}\left(s, x_{s}^{t, y, v}\right), \varphi(y)\right) d y \\
\int_{R^{d}}(g(x), \varphi(T, x)) d x=\int_{R^{d}}\left(g\left(x_{T}^{t, y, v}\right), \varphi(y)\right) d y \\
\int_{R^{d}} \int_{s}^{T}\left(f\left(r, x, V(r, x), \sigma^{*} \nabla V(r, x), v_{r}\right), \varphi(r, x)\right) d r d x \\
=\int_{R^{d}} \int_{s}^{T}\left(f\left(r, x_{r}^{t, y, v}, V\left(r, x_{r}^{t, y, v}\right),\left(\sigma^{*} \nabla V\right)\left(r, x_{r}^{t, y, v}\right), v_{r}\right), \varphi(y)\right) d r d y \\
\int_{R^{d}} \int_{s}^{T}\left(\left(\sigma^{*} \nabla \bar{V}\right)(r, x), \varphi(r, x)\right) d W_{r} d x=\int_{R^{d}} \int_{s}^{T}\left(\left(\sigma^{*} \nabla \bar{V}\right)\left(r, x_{r}^{t, y, v}\right), \varphi(y)\right) d W_{r} d y .
\end{gathered}
$$

So (82) becomes

$$
\begin{aligned}
& \int_{R^{d}} \bar{V}\left(s, x_{s}^{t, y, v}\right) \varphi(y) d y \\
\geq & \int_{R^{d}} g\left(x_{T}^{t, y, v}\right) \varphi(y) d y \\
& +\int_{s}^{T} \int_{R^{d}}\left(f\left(r, x_{r}^{t, y, v}, \bar{V}\left(r, x_{r}^{t, y, v}\right),\left(\sigma^{*} \nabla \bar{V}\right)\left(r, x_{r}^{t, y, v}\right), v_{r}\right), \varphi(y)\right) d y d r \\
& -\int_{s}^{T} \int_{R^{d}}\left(\left(\sigma^{*} \nabla \bar{V}\right)\left(r, x_{r}^{t, y, v}\right), \varphi(y)\right) d y d W_{r} .
\end{aligned}
$$


Since $\varphi$ is arbitrary, we have proven that for $\rho(y) d y$ almost every $y$,

$$
\begin{aligned}
\bar{V}\left(s, x_{s}^{t, y, v}\right) \geq & g\left(x_{T}^{t, y, v}\right)+\int_{s}^{T} f\left(r, x_{r}^{t, y, v}, \bar{V}\left(r, x_{r}^{t, y, v}\right),\left(\sigma^{*} \nabla \bar{V}\right)\left(r, x_{r}^{t, y, v}\right), v_{r}\right) d r \\
& -\int_{s}^{T}\left(\sigma^{*} \nabla \bar{V}\right)\left(r, x_{r}^{t, y, v}\right) d W_{r} .
\end{aligned}
$$

Let

$$
\begin{aligned}
\bar{y}_{s}^{t, y, v}:= & g\left(x_{T}^{t, y, v}\right)+\int_{s}^{T} f\left(r, x_{r}^{t, y, v}, \bar{V}\left(r, x_{r}^{t, y, v}\right),\left(\sigma^{*} \nabla \bar{V}\right)\left(r, x_{r}^{t, y, v}\right), v_{r}\right) d r \\
& -\int_{s}^{T}\left(\sigma^{*} \nabla \bar{V}\right)\left(r, x_{r}^{t, y, v}\right) d W_{r} .
\end{aligned}
$$

Then

$$
\begin{aligned}
\bar{V}\left(s, x_{s}^{t, y, v}\right)= & g\left(x_{T}^{t, y, v}\right)+\int_{s}^{T} f\left(r, x_{r}^{t, y, v}, \bar{V}\left(r, x_{r}^{t, y, v}\right),\left(\sigma^{*} \nabla \bar{V}\right)\left(r, x_{r}^{t, y, v}\right), v_{r}\right) d r \\
& +\left(\bar{V}\left(s, x_{s}^{t, y, v}\right)-\bar{y}_{s}^{t, y, v}\right)-\int_{s}^{T}\left(\sigma^{*} \nabla \bar{V}\right)\left(r, x_{r}^{t, y, v}\right) d W_{r} .
\end{aligned}
$$

Where $\bar{V}\left(s, x_{s}^{t, y, v}\right)-\bar{y}_{s}^{t, y, v} \geq 0$, so by the comparison theorem of the BSDE, we know that the $\bar{V}\left(r, x_{r}^{t, y, v}\right)$ is the g-supersolution of the BSDE (48). We get

$$
\bar{V}\left(s, x_{s}^{t, y, v}\right) \geq Y_{s}^{s, x_{s}^{t, y, v}, v} .
$$

Let us make the same change of variable $y=\widehat{x}_{r}^{t, x, v^{\prime}}$ in each term of (84), so (84) becomes

$$
\begin{aligned}
& \int_{R^{d}} \bar{V}\left(s, x_{s}^{t, y, v^{\prime}}\right) \varphi(y) d y-\varepsilon \\
\leq & \int_{R^{d}} g\left(x_{T}^{t, y, v^{\prime}}\right) \varphi(y) d y \\
& +\int_{s}^{T} \int_{R^{d}}\left(f\left(r, x_{r}^{t, y, v^{\prime}}, \bar{V}\left(r, x_{r}^{t, y, v^{\prime}}\right),\left(\sigma^{*} \nabla \bar{V}\right)\left(r, x_{r}^{t, y, v^{\prime}}\right), v_{r}^{\prime}\right), \varphi(y)\right) d y d r \\
& -\int_{s}^{T} \int_{R^{d}}\left(\sigma^{*} \nabla \bar{V}\right)\left(r, x_{r}^{t, y, v^{\prime}}\right) \varphi(y) d y d W_{r} .
\end{aligned}
$$

Since $\varphi$ is arbitrary, we have proven that for $\rho(y) d y$ almost every $y$,

$$
\begin{aligned}
& \bar{V}\left(s, x_{s}^{t, y, v^{\prime}}\right)-\frac{\varepsilon}{\int_{R^{d}} \varphi(y) d y} \\
\leq & g\left(x_{T}^{t, y, v^{\prime}}\right)+\int_{s}^{T} f\left(r, x_{r}^{t, y, v^{\prime}}, \bar{V}\left(r, x_{r}^{t, y, v^{\prime}}\right),\left(\sigma^{*} \nabla \bar{V}\right)\left(r, x_{r}^{t, y, v^{\prime}}\right), v_{r}^{\prime}\right) d r \\
& -\int_{s}^{T}\left(\sigma^{*} \nabla \bar{V}\right)\left(r, x_{r}^{t, y, v^{\prime}}\right) d W_{r} .
\end{aligned}
$$


We let $\widetilde{V}\left(s, x_{s}^{t, y, v^{\prime}}\right)=\bar{V}\left(s, x_{s}^{t, y, v^{\prime}}\right)-\frac{\varepsilon}{\int_{R^{d}} \varphi(y) d y}$, then we get

$$
\begin{aligned}
& \widetilde{V}\left(s, x_{s}^{t, y, v^{\prime}}\right)+\frac{\varepsilon}{\int_{R^{d}} \varphi(y) d y} \\
\leq & g\left(x_{T}^{t, y, v^{\prime}}\right) \\
& +\int_{s}^{T} f\left(r, x_{r}^{t, y, v^{\prime}}, \widetilde{V}\left(r, x_{r}^{t, y, v^{\prime}}\right)+\frac{\varepsilon}{\int_{R^{d}} \varphi(y) d y},\left(\sigma^{*} \nabla \widetilde{V}\right)\left(r, x_{r}^{t, y, v^{\prime}}\right), v_{r}^{\prime}\right) d r \\
& -\int_{s}^{T}\left(\sigma^{*} \nabla \widetilde{V}\right)\left(r, x_{r}^{t, y, v^{\prime}}\right) d W_{r}+\frac{\varepsilon}{\int_{R^{d}} \varphi(y) d y} .
\end{aligned}
$$

Define

$$
\begin{aligned}
& K^{t, y, v^{\prime}}:=g\left(x_{T}^{t, y, v^{\prime}}\right) \\
+ & \int_{s}^{T} f\left(r, x_{r}^{t, y, v^{\prime}}, \widetilde{V}\left(r, x_{r}^{t, y, v^{\prime}}\right)+\frac{\varepsilon}{\int_{R^{d}} \varphi(y) d y},\left(\sigma^{*} \nabla \widetilde{V}\right)\left(r, x_{r}^{t, y, v^{\prime}}\right), v_{r}^{\prime}\right) d r \\
& -\int_{s}^{T}\left(\sigma^{*} \nabla \widetilde{V}\right)\left(r, x_{r}^{t, y, v^{\prime}}\right) d W_{r}+\frac{\varepsilon}{\int_{R^{d}} \varphi(y) d y} .
\end{aligned}
$$

By (90), we can know that

$$
\begin{aligned}
& \widetilde{V}\left(s, x_{s}^{t, y, v^{\prime}}\right)+\frac{\varepsilon}{\int_{R^{d}} \varphi(y) d y} \\
= & g\left(x_{T}^{t, y, v^{\prime}}\right)-\left(K^{t, y, v^{\prime}}-\widetilde{V}\left(s, x_{s}^{t, y, v^{\prime}}\right)-\frac{\varepsilon}{\int_{R^{d}} \varphi(y) d y}\right) \\
& +\int_{s}^{T} f\left(r, x_{r}^{t, y, v^{\prime}}, \widetilde{V}\left(r, x_{r}^{t, y, v^{\prime}}\right)+\frac{\varepsilon}{\int_{R^{d}} \varphi(y) d y},\left(\sigma^{*} \nabla \widetilde{V}\right)\left(r, x_{r}^{t, y, v^{\prime}}\right), v_{r}^{\prime}\right) d r \\
& -\int_{s}^{T}\left(\sigma^{*} \nabla \widetilde{V}\right)\left(r, x_{r}^{t, y, v^{\prime}}\right) d W_{r}+\frac{\varepsilon}{\int_{R^{d}} \varphi(y) d y} .
\end{aligned}
$$

Because $K^{t, y, v^{\prime}}-\widetilde{V}\left(s, x_{s}^{t, y, v^{\prime}}\right)-\frac{\varepsilon}{\int_{R^{d}} \varphi(y) d y} \geq 0$, so by the comparison theorem of the BSDE, we can get

$$
\widetilde{V}\left(s, x_{s}^{t, y, v^{\prime}}\right)+\frac{\varepsilon}{\int_{R^{d}} \varphi(y) d y} \leq Y_{s}^{s, x_{s}^{t, y, v^{\prime}}, v^{\prime}}+\frac{\varepsilon}{\int_{R^{d}} \varphi(y) d y} .
$$

So

$$
\bar{V}\left(s, x_{s}^{t, y, v^{\prime}}\right) \leq Y_{s}^{s, x_{s}^{t, y, v^{\prime}}, v^{\prime}}+\frac{\varepsilon}{\int_{R^{d}} \varphi(y) d y} .
$$

Combining (88) with (92), we know that

$$
\bar{V}(t, y)=\sup _{v \in \mathcal{U}} Y_{t}^{t, y, v}
$$

Then $\bar{V}(t, y)$ is also the value of $\sup _{v \in \mathcal{U}} J(t, y, v)$, from uniqueness of the solution of cost functional and the uniqueness of supremum, we get uniqueness of weak solution for PDEs (51), and $\bar{V}(t, x)=V(t, x)$. 


\section{References}

[1] V. Bally, A. Matoussi, Weak solutions for SPDEs and backward doubly stochastic differential equations, Journal of Theoretical Probability, 14 (2001), pp. 125-164, .

[2] G. Barles, R. Buckdahn, E. Pardoux, Backward stochastic differential equations and integral-partial differential equations. Stochastics Stochastics Rep., 60 (1997), pp. 57-83.

[3] M. G. Crandall, H. Ishii, P. L. Lions, User's guide to viscosity solution of second order partial differential equations, Bull. Amer. Math. Soc. (N.S), 27 (1992), PP. 1-67.

[4] D. Duffie \& L. Epstein, Stochastic differential utility, Econometrica, 60 (1992), pp. 353-394.

[5] N. Ikeda, S. Watanabe, Stochastic differential equations and diffusion process, NorthHolland Press, Kondansha, (1989).

[6] H. Kunita, Stochastic flow acting on schwartz distributions, J. Theor Prob., 7(2)(1994), pp. $247-278$, .

[7] N. El Karoui, S. Peng \& M. C. Quenez, Backward Stochastic Differential Equation in Finance, Math. Finance, 7 (1997), pp. 1-71.

[8] B. Mezerdi, S. Bahlali, Approximation in optimal control of diffusion processes, Random Oper. Stochastic Equations, 8 (2000), pp. 365-372.

[9] B. Mezerdi, S. Bahlali, Necessary conditions for optimality in relaxed stochastic control problems, Stochastics Stochastics Rep., 73 (2002), pp. 201-218.

[10] M. N'Zi, Y. Ouknine, and A. Sulem, Regularity and representation of viscosity solutions of partial differential equations via backward stochastic differential equations.Stochastic Processes and Their Applications, 116 (2006), PP. 1319-1339.

[11] Y. Ouknine, I. Turpin, Weak solutions of semilinear PDEs in sobolev spaces and their probabilistic interpretation via the FBSDEs. Stochastic Analysis and Applications, 24 (2006), pp. 871-888.

[12] E. Pardoux \& S. Peng, Adapted solution of a backward stochastic differential equation, Systems and Control Letters, 14 (1990), pp. 55-61.

[13] S. Peng, A generalized dynamic programming principle and Hamilton-Jacobi-Bellmen equation, Stochastics Stochastics Rep., 38(1992), pp. 119-134.

[14] S. Peng, Backward stochastic differential equations-stochastic optimization theory and viscosity solutions of HJB equations, in Topics on Stochastic Analysis, J.Yan, S.Peng, S.Fang and L.Wu, eds., Science Press, Beijing, 1997, pp. 85-138 (in Chinese).

[15] S. Peng, Monotonic limit throrem of BSDE and nonlinear decomposition theorem of DoobMeyer's type, Probability Theory Relat. Fields, 113 (1999), pp. 473-499.

[16] Z. Wu, Z. Yu, Dynamic programming principle for one kind of stochastic recursive optimal control problem and Hamilton-Jacobi-Bellman equation. SIAM J.Control Optim, 47 (2008), pp. 2616-2641. 
[17] J. Yong, X. Y. Zhou, Stochastic Control, Hamilton Systems and HJB Equations, Appl. Math., 43, Springer-Verlag, New York, 1999.

[18] Q. Zhang, H. Z. Zhao, Stationary solution of SPDEs and infinite horizon BSDEs. Journal of Functional Analysis, 252 (2007), pp. 171-219. 